Journées Mixtes de la Société Française de Toxicologie Analytique \& de la Société Marocaine de Toxicologie Clinique et Analytique

Société Française de Toxicologie Analytique \& Société Marocaine de Toxicologie Clinique et Analytique. Joint Meeting

Marrakech (Maroc), 9-11 novembre 2006

Résumés des communications orales

- Toxicologie clinique

- Toxicologie médico-légale

- Toxicologie analytique

- Résultats de toxicologie et prise de décision

- Thèmes libres

Résumés des communications affichées 


\section{COMMUNICATIONS ORALES}

\section{Toxicologie clinique}

\section{Intoxication par le redoul (Coriaria myr- tifolia) : une nouvelle observation et revue de la littérature}

\section{J. ARDITTI, L. TICHADOU, M. HAYEK-LANTHOIS, L. DE HARO \\ Centre Antipoison, Hôpital Salvator, 13009 Marseille, France}

Introduction : le redoul (Coriaria myrtifolia) est un arbuste de 2 à 3 mètres qui doit être considéré comme une des plantes les plus neurotoxiques d'Europe de l'ouest et d'Afrique du nord. Cette espèce produit une toxine, la coriamyrtine, qui est présente à hautes concentrations dans les fruits. L'ingestion de quelques fruits peut induire des troubles digestifs et surtout neurologiques incluant convulsions, coma et apnée (1).

Cas clinique : les auteurs rapportent un nouveau cas s'étant déroulé dans la région de Perpignan. Il s'agit d'un enfant de 8 ans sans antécédent qui a ingéré plusieurs poignées de fruits d'un arbuste sauvage. Une heure après ce repas, l'enfant a présenté des vomissements noirâtres et des vertiges. Ce garçon est alors orienté vers l'hôpital où dès son arrivée il présente des convulsions traitées par diazépam. Malgré un retour à la normale rapide, l'enfant a présenté 2 heures plus tard un nouvel épisode de convulsions et de vomissement. L'évolution a été caractérisée par une guérison complète dès le lendemain de l'ingestion.

Discussion : afin de mieux caractériser les conséquences de l'intoxication par le redoul, 83 cas de la littérature ont été analysés. Les cas sont uniquement observés dans 3 pays (Espagne, France et Maroc) ; le délai moyen d'apparition des symptômes a été de 150 minutes et les signes cliniques les plus fréquents sont des troubles neurologiques à type de convulsions voire d'état de mal épileptique. A noter que parmi ces 83 patients, 11 sont décédés ( 6 enfants et 5 adultes) principalement dans des descriptions anciennes.

Conclusion : les fruits du redoul ont un goût agréable et un aspect qui peut être à l'origine de confusion avec des mûres, d'où la possibilité d'observer des cas d'ingestion de grandes quantités de baies. Il s'agit d'une espèce neurotoxique qui peut être impliquée dans des cas d'intoxications graves avec éventuelle mise en jeu du pronostic vital.

\section{Références :}

1. De Haro L. et coll. Poisoning by Coriaria myrtifolia L.: a new case report and review of the literature. Toxicon 2005 ; 46(6) : 600-3.

2. Skalli S. et coll. Intoxication aiguë par le redoul (Coriaria myrtifolia) : trois observations. Presse Méd. 2002; 31 : 1554-6.

\section{Manifestations cardiovasculaires de l'in- toxication au phosphure d'aluminium}

$\underline{\text { S. ACHOUR }}{ }^{(1)}$, EL M. AKKAOUI (2), K.H. ABIDI ${ }^{(2)}$, N. MADANI ${ }^{(2)}$, R. ABOUQAL ${ }^{(2)}$, ZEGGOUAGH $^{(2)}$

(1) Centre Antipoison et de Pharmacovigilance du Maroc ;

(2) Service de réanimation médicale et de toxicologie clinique, CHU Avicenne, Rabat, Maroc.

Objectif : l'intoxication aiguë au phosphure d'aluminium (PAL) est responsable d'une lourde mortalité liée essentiellement à l'atteinte cardiaque et aux perturbations hémodynamiques graves induites par ce produit très toxique (1). Le but de nos observations était de décrire l'atteinte cardiaque et les anomalies observées à l'échocardiographie compliquant l'intoxication par le PAL.

Observation 1 : Mme S.S, âgée de 19 ans, avait ingéré de façon accidentelle trois comprimés d'un raticide grisâtre mélangé initialement aux aliments destinés pour la dératisation. La patiente a présenté immédiatement des douleurs abdominales, des vomissements alimentaires, des sueurs profuses et une tachycardie à 110 battements par minute, la radiographie pulmonaire était normale et le bilan biologique était sans anomalies, l'électrocardiogramme à l'admission a montré un susdécalage du segment ST en V1 et V2, et le prélèvement toxicologique dans le sang était positif au PAL. Le traitement était basé sur un lavage gastrique et l'administration de drogues vasoactives à type de dobutamine assurant une stabilité hémodynamique.

Observation 2 : Mr M.H., âgé de 28 ans, avai ingéré dans un but d'autolyse un comprimé de phostoxin dissous dans de l'eau. Deux heures après l'ingestion, il a présenté des vomissements incoercibles, une hypotension $(\mathrm{TA}=11 / 06)$, une tachycardie à 108 batt. /min, le reste de l'examen somatique était sans particularités. La radiographie pulmonaire était normale, l'électrocardiogramme effectué à l'admission montrait un sus décalage ST en V2, V3 V4 avec des ondes T amples, le bilan biologique a révélé un taux de prothrombine diminué à $43 \%$, une troponine à $37,5 \mathrm{ug} / \mathrm{mL}$, une $\mathrm{CPK}$ à $1110 \mathrm{IU} / \mathrm{L}$ et une LDH à 1010IU/L. Le prélèvement toxicologique était positif dans le sang au PAL, l'hypotension était jugulée par un remplissage sans avoir recours aux drogues vasoactives. L'échocardiographie transthoracique réalisée dans les deux cas avait révélé de façon semblable une atteinte myocardique diffuse et avec hypokinésie sévère du ventricule gauche, les fractions d'éjection du ventricule gauche (FEVG) ont été respectivement $20 \%$ chez la femme et de $30 \%$ chez l'homme, l'échocardiographie de contrôle avait révélé dans les deux cas une amélioration de la contractilité myocardique au bout de huit jours.

Conclusion : dans l'intoxication par le PAL, l'atteinte cardiaque réversible est peu décrite (2). L'échocardiographie réalisée chez nos deux patients avait permis de documenter sa réversibilité. 


\section{Références :}

1. Singh S. et coll. Aluminum phosphide ingestion - a clinico-pathologic study: J. Toxicol Clin toxicol. 1996 ; 34(6) : 703-6.

2. Gupta M.S. et coll. Cardiovascular manifestaions in aluminium phosphide poisoning with special reference to echocardiographic changes: J. Assoc. Physicians India. 1995; 43(11): 773-4, 779-80.

3. Sing R.B. et coll. Cardiovascular manifestations of aluminium phosphide intoxication: J. Assoc Physicians India. 1989; 37(9) : 590-2.

\section{L'usage des antidotes en préhospitalier}

\section{ALAOUI MOUSTAIN}

SAMU, Gendarmerie Royale, Salé, Maroc

Introduction : la définition la plus claire d'un antidote est probablement celle qu'en donne F. Baud : « l'antidote est un médicament ou un dispositif médical dont l'action spécifique a pu être établie chez l'animal et chez l'homme, capable soit de modifier la cinétique du toxique, soit d'en diminuer les effets au niveau de récepteurs ou de cibles spécifiques, et dont l'utilisation améliore le pronostic fonctionnel ou vital de l'intoxication ». En préhospitalier et aux services d'accueil des urgences, la primauté des soins revient aux traitements symptomatiques cardio-vasculaire, respiratoire et neurologique "Traiter le patient avant de traiter le toxique" (Goldfrank). Les traitements antidotes, lorsqu'ils sont disponibles, doivent être mis en route le plus tôt possible car ils peuvent constituer le traitement salvateur de nombreuses intoxications. Le retard à l'administration peut constituer une perte de chance majeure. Toutefois, La difficulté est certaine pour les antidotes d'exception, indispensables mais d'indications exceptionnelles, d'un coût élevé ou d'un délai de péremption court, il n'est pas réaliste d'envisager leur disponibilité dans tous les SMUR. Les traitements évacuateurs ont peu de place en préhospitalier et ne doivent pas retarder le transfert du patient à l'hôpital.

Les antidotes : du fait de leur remarquable efficacité thérapeutique, certains traitements antidotiques méritent de voir leur place soulignée en préhospitalier :

- L'immunotoxicothérapie par le fragment Fab a largement simplifié la prise en charge des intoxications par les digitaliques. Ses modalités d'utilisation (neutralisation curative ou préventive) doivent être connues du plus grand nombre des urgentistes afin de ne pas retarder la mise en oeuvre d'un traitement simple et remarquablement efficace (1).

- L'hydroxocobalamine. La responsabilité des cyanures est désormais bien établie dans la mortalité constatée chez les victimes exposées aux fumées d'incendie. L'hydroxocobalamine possède dans cette situation un fort pouvoir antidotique. Son efficacité dépend étroitement de la précocité de son administration, qui devra être débutée sur les lieux mêmes de l'intoxication lorsque l'intoxication apparaît probable (2).

- La N-acétylcystéïne (NAC). Les mécanismes de toxicité du paracétamol permettent de mieux comprendre les effets bénéfiques potentiels du traitement antidotique par la NAC. La démonstration de l'existence d'une relation claire entre concentration sanguine de paracétamol et risque hépatotoxique s'ajoute à l'individualisation récente de facteurs de gravité (dénutrition, éthylisme, co-intoxication par un médicament inducteur), permettant ainsi de mieux définir les indications du traitement antidotique par la NAC (2).

- Le flumazénil. Il a totalement modifié l'approche diagnostique et thérapeutique de l'intoxication par benzodiazépines. Ses modalités pratiques d'utilisation sont clairement établies mais son utilisation doit faire l'objet d'une réflexion systématique à la recherche d'une éventuelle contre-indication (risque convulsif), même si des données récentes plaident en faveur de son innocuité lors des intoxications polymédicamenteuses (2).

- Le fomépizole (4-méthyl-pyrazole). C'est un puissant antidote de l'éthylène glycol. Il représente un progrès très important dans les modalités de traitement de ce type d'intoxication. Sa place dans le traitement de l'intoxication par le méthanol reste encore à déterminer (3).

\section{Références :}

1. Bismuth C. et coll. Immunotoxicothérapie. Rev. Prat. $1997 ; 47: 754-759$.

2. Baud F. et coll. Mécanisme d'action, indications et modalités d'administration des antidotes. Intoxications aiguës. Elsevier 1999 ; 179-194.

3. Kupferschmidt H. Antidotes contre les intoxications Bull. Méd. Suisse 2005 ; 86 : 12.

\section{Les intoxications mortelles, accidentelles et suicidaires chez l'adolescent au Maroc (1992-2005)}

\section{A. KHATTABI, Z. SGHIER, N. RHALEM,} I. SEMLALI, A. SOULAYMANI, R. SOULAYMANI

Centre Anti Poison et de Pharmacovigilance du Maroc, Rabat, Maroc

Objectif : décrire le profil épidémiologique des intoxications mortelles chez les adolescents au Maroc.

Méthode : analyse descriptive des intoxications mortelles chez les personnes âgées de 10 à 19 ans au Maroc. Les données sont recueillies à partir des fiches de déclaration des intoxications présumées ou avérées du centre National Anti Poison du Maroc (CAPM) du $1^{\text {er }}$ janvier 1992 au 31 décembre 2005. La saisie et l'analyse sont réalisées sur Epi info 2002.

Résultats : durant la période d'étude, le CAPM a reçu 89155 cas de déclarations d'intoxications. Parmi ces cas, nous avons enregistré 194 intoxications mortelles chez l'adolescent, dont 88 étaient des suicides et 82 étaient des décès accidentels. Les victimes étaient le 
plus souvent de sexe féminin (70\%). L'intoxication était la méthode de suicide prédominante chez les filles (60\%) que chez les garçons (40\%). L'intoxication fatale est souvent accidentelle entre 10 à 14 ans $(70.5 \%)$ qu'entre 15 à 19 ans (29.5\%). Cependant, l'intoxication était le plus souvent la méthode de suicide chez les adolescents entre 15 et 19 ans (91\%). Le taux de mortalité par intoxication était plus élevé dans la tranche 15 à 19 ans $(1.3 \%$ ) que dans la tranche 10 - à 14 ans $(0.8 \%$ ) . La distribution des substances impliquées était différente pour 10 à 14 ans comparées à 15 à 19 ans et pour les suicides comparés aux accidents. Dans la tranche 10 à 14 ans, les envenimations ont expliqué $44.5 \%$ des décès accidentelles. Les plantes ont provoqué 20.4\% des décès accidentels suivies par le monoxyde de carbone $(11.1 \%)$. Les pesticides, étaient impliqués dans $71.4 \%$ des suicides et seulement dans $9.3 \%$ des décès accidentelles. Dans la tranche 15 à 19 ans, les pesticides ont été impliqués dans $49.5 \%$ des suicides et $22 \%$ des décès accidentels. La paraphènylène diamine (PPD) était utilisée dans $28.6 \%$ des suicides chez les deux tranches d'âge.

Conclusions : le décès dû aux intoxications accidentelles et suicidaires chez l'adolescent est un problème de santé publique exigeant une intervention adéquate parce que, derrière chaque mort il y a beaucoup plus d'adolescents qui s'intoxiquent et survivent. L'analyse des données de fatalité a montré que les taux de suicides et de décès accidentels par intoxication étaient élevés chez les filles de 15 à 19 ans contrairement à d'autres études $(1,2)$ où les victimes sont le plus souvent de sexe masculin. Notre étude a donc permis l'identification du groupe de population à haut risque où les efforts d'éducation et de sensibilisation pourraient converger pour diminuer la mortalité chez l'adolescent.

\section{Références :}

1. Shepherd G. et coll. Accidental and suicidal adolescent poisoning deaths in the United States, 1979-1994. Arch. Pediatr. Adolesc. Med. 1998 ; 152 : 1181-1185.

2. Targosz D. et coll. Frequency and pattern of poisoning in adult and adolescent Krakow population in 2003 and 1983. Przegl Lek. 2005 ; 62(6) : 446-52.

\section{Intoxications volontaires par le mépro- bamate : suivi clinique et analytique des observations colligées au cours du pre- mier trimestre 2006}
I. BLANC, L. TICHADOU, J.H. BOURDON, L. DE HARO, M. HAYEK, J. ARDITTI

Centre Antipoison, Hôpital Salvator, Marseille, France

Introduction : le méprobamate est un anxiolytique de la famille des carbamates d'utilisation très répandue. Il est utilisé seul ou associé à une phénothiazine dans le cadre du traitement de l'insomnie et de l'aide au sevrage alcoolique. Le centre antipoison de Marseille est fré- quemment sollicité $(7.8 \%$ des intoxications volontaires) pour la prise en charge d'intoxications volontaires. Les toxicologues se doivent d'attirer l'attention des cliniciens sur la gravité potentielle, notamment cardiovasculaire de cette intoxication.

Objectif : identifier une relation entre les concentrations sanguines, la dose supposée ingérée (DSI) et le tableau clinique afin de cibler les patients à risque de complication majeure lors d'intoxications volontaires.

Méthodes : le recueil des dossiers de janvier à juin 2006 a été effectué à partir des dossiers de l'unité de réponse téléphonique en urgence (96), des dossiers de patients pour lesquels un dosage sanguin de méprobamate a été réalisé au laboratoire de toxicologie du centre antipoison (28) Les dossiers médicaux ont été ensuite demandés aux services d'urgence et réanimation concernés et des dossiers communs aux 2 sources (17). Les données ont été saisies dans la base Access ${ }^{\circledR}$ puis exploitées avec Excel®.

Résultats : notre série comporte 124 patients, 78 femmes et 46 hommes d'âge moyen 40,6 ans. Les spécialités concernées sont pour $56 \%$ la Mepronizine ${ }^{\circledR}$ et pour $33 \%$ l'Equanil ${ }^{\circledR} 400$, la source de méprobamate étant inconnue des 10\% des cas. Dans 93 cas le méprobamate est associé à d'autres substances psychoactives (benzodiazépines, neuroleptiques, alcool). Nous avons établi 2 sous groupes afin d'étudier d'une part les relations entre DSI et tableau clinique et d'autre part dosage du méprobamate et tableau clinique. Dans le $1^{\text {er }}$ sous-groupe, pour des DSI inférieures à 4 grammes, 38 patients sont somnolents, 4 présentent un coma, 10 une hypotension et il n'y a pas de collapsus. Seuls 5 patients présentent des complications. Pour des DSI comprises entre 4 et 11 grammes, 13 patients sur 20 ont une somnolence, 6 comas, 2 collapsus, un patient est décédé. Pour des DSI supérieures à 11 grammes et pour des DSI inconnues, on note 20 comas, 9 collapsus, 3 pneumopathies d'inhalation, 1 décès, 1 cas de rhabdomyolyse. Dans le $2^{\text {eme }}$ sous-groupe, les 5 patients ayant présenté un collapsus ont eu des concentrations supérieures à $94 \mathrm{mg} / \mathrm{L}$. C'est aussi dans cette zone de concentration que l'on retrouve 1 décès, 4 pneumopathies et 2 rhabdomyolyses. Les cas les graves et l'imputabilité du méprobamate dans les décès survenus dans la population étudiée seront étudiés.

Conclusion : dans cette série de 124 observations, il apparaît difficile d'établir, dans tous les cas, une corrélation clinico-biologique significative. En effet, comme dans toutes les études impliquant une population de patients suicidaires, de nombreux paramètres inconnus et/ou aléatoires compliquent l'interprétation des résultats. Cependant, des concentrations sanguines de méprobamate supérieures à $50 \mathrm{mg} / \mathrm{L}$ sont retrouvées chez $50 \%$ des patients présentant des signes cliniques nécessitant une surveillance spécialisée, une concentration supérieure à $80 \mathrm{mg} / \mathrm{L}$ est un critère de gravité pour le patient. 


\section{Tentatives suicidaires aux médicaments F. ABOUALI, N. RHALEM, R. SOULAYMANI- BENCHEIKH \\ Centre Anti Poison et de Pharmacovigilance du Maroc, Rabat, Maroc}

Objectif : montrer l'importance des intoxications médicamenteuses dans un but suicidaire, tirer les caractéristiques épidémiologiques de cette pathologie et analyser l'effet des différents facteurs pouvant influencer l'évolution.

Méthode : il s'agit d'une étude rétrospective portant sur les cas de tentatives suicidaires par médicaments parvenus par téléphone à l'unité d'information toxicologique du centre antipoison et de pharmacovigilance du Maroc durant 3 ans (2003 à 2005), et ayant nécessité soit un avis spécialisé pour la prise en charge soit une orientation.

Résultats : au total 595 cas de tentatives suicidaires ont été recensés. Ces tentatives sont particulièrement fréquentes chez l'adolescent et l'adulte jeune (age moyen de $20.90 \pm 9.19$ ans) avec une prédominance féminine (sexe ratio de 0.35). La majorité des appels sont parvenus d'un personnel de santé (93\%) exerçant dans un centre hospitalier universitaire dans $50 \%$. Le maximum de tentatives suicidaires a été enregistré en fin de journée. Les spécialités médicamenteuses neuropsychiatriques ont été à l'origine de la majorité des cas (43.9\%) et la classe des benzodiazépines a la portion la plus importante par rapport à l'ensemble des classes des médicaments $(24.7 \%)$, le bromazepam étant le principe actif le plus retrouvé (10.6\%). Les tentatives de suicide se font exclusivement par voie orale et à domicile. Le tableau clinique est prédominé par la symptomatologie neurologique. L'évolution a été marquée par 8 cas de décès $(1.4 \%)$.

Conclusion : les résultats de l'étude statistique montrent que les tentatives suicidaires aux médicaments constituent une urgence fréquente nécessitant une prise en charge multidisciplinaire avec une action particulière du psychiatre. Certains facteurs de gravité peuvent être démontrés (âge, sexe, classe du médicament...). La stratégie globale de prévention nécessite l'intervention de plusieurs domaines (social, économique, politique et médical) et une prise en charge adaptée et réfléchie des suicidants en collaboration avec les services des urgences et de réanimation.

\section{Toxicologie médico-légale}

\section{Relations entre conclusions d'autopsie et résultats toxicologiques}

J.C. ALVAREZ ${ }^{(1)}$, C. DUVERNEUIL-MAYER ${ }^{(1)}$, E. $\mathrm{ABE}^{(1)}$, G. DE LA GRANDMAISON ${ }^{(2)}$, F. PARAIRE ${ }^{(2)}$, C. RAMBAUD ${ }^{(2)}$, P. DE MAZANCOURT ${ }^{(1)}$, M. DURI$\mathrm{GON}^{(2)}$

(1) Laboratoire de Toxicologie ;

(2) Médecine Légale, CHU Poincaré, Garches, France. Objectif : analyser les résultats d'analyses toxicologiques réalisées lors de 550 autopsies et 149 levées de corps pour recherche des causes de décès et comparer ces résultats toxicologiques aux conclusions macroscopiques de l'autopsie.

Méthodes : une recherche urinaire des stupéfiants (amphétamines, cannabis, cocaïne, opiacés, méthadone, buprénorphine, LSD) est réalisé par immunoanalyse. Tous les échantillons positifs sont dosés en CPG-SM ou LC-SM/SM dans le sang et l'urine. Des recherches non spécifiques ("screening") après extraction liquide/liquide en milieu acide et basique et des recherches spécifiques des psychotropes (barbituriques, benzodiazépines, antidépresseurs et neuroleptiques) sont effectuées par CPG-SM, CLHP-BD et CLHP-SM/SM. L'alcoolémie est dosée par CPG-FID. L'HbCO est dosée sur un hémoxymètre $\mathrm{ABL}$ 700. Le lithium est recherché par photomètrie de flamme. D'autres recherches plus spécifiques peuvent être réalisées en fonction du contexte (substances volatiles notamment).

Résultats : la présence d'un xénobiotique est retrouvée dans 589 des 699 autopsies ou levées de corps (84\%). Une origine toxique du décès est conclue dans 195 cas, soit 27,9\% des 699 décès. Parmi les 550 décès pour lesquels une autopsie a été réalisée, 133 cas concluaient à une origine toxique probable du décès. 114 cas $(85 \%)$ ont été confirmés par l'analyse toxicologique, 19 cas $(15 \%)$ n'ayant pas montré de concentrations toxiques en xénobiotiques. Parmi les 343 autopsies pour lesquelles l'origine du décès était connue et à priori non toxique (pendaison, noyades...), 91 cas $(26,5 \%)$ ont révélé la présence de substance pouvant avoir un rapport direct ou indirect avec la cause connue du décès et dans 14 cas (4\%), le décès pouvait même s'expliquer par la présence seule de toxiques. Dans 31 cas, la cause du décès était à priori naturelle, mais un doute persistait, l'analyse toxicologique révélant un décès toxique dans 13 cas $(41,9 \%) .43$ autopsies concluaient à une mort naturelle, toutes confirmées par l'absence de toxique. 54 des 149 prélèvements réalisés lors d'une levée de corps $(36,2 \%)$ ont permis de conclure à une origine toxique du décès, évitant ainsi l'autopsie.

Dans 383 cas (65\% des positifs), on retrouve au moins un médicament et dans 138 cas (23\%), au moins un stupéfiant. Les traitements de substitution aux opiacés 
(méthadone $\mathrm{n}=17$, buprénorphine $\mathrm{n}=14$ ou les deux $\mathrm{n}=2)$ sont retrouvés dans 33 cas $(5,6 \%)$. L'alcool est présent dans 326 cas $(55 \%)$, dont 36 cas $>2 \mathrm{~g} / \mathrm{l}$, et une $\mathrm{HbCO}$ supérieure à $17 \%$ est retrouvée dans 19 cas $(3,2 \%)$. On retrouve des benzodiazépines dans 241 cas (41\%), essentiellement du diazépam, nordiazépam, oxazépam et bromazépam, des antidépresseurs dans 125 cas $(21 \%)$, essentiellement de la clomipramine, fluoxétine et citalopram, des neuroleptiques dans 99 cas $(17 \%)$, essentiellement de la cyamémazine, alimémazine et acéprométazine et des $\beta$-bloquants dans 21 cas $(3,5 \%)$. Paracétamol, méprobamate, dextropropoxyphène, acide valproïque, tramadol et atracurium sont les autres médicaments les plus souvent retrouvés. Les barbituriques sont retrouvés dans 10 cas (phénobarbital $=9$, thiopental $=1$ ). Le cannabis est le stupéfiant le plus souvent rencontré $(\mathrm{n}=90)$, suivi de la morphine $(n=39)$ et de l'héroïne $(n=25$, avec présence de 6monoacétylmorphine). La strychnine, la chloroquine, la colchicine, le dichlorvos (organophosphoré), la crimidine (souricide), l'embutramide (euthanasiant) et le White Spirit ont été retrouvés chacun dans un cas.

Conclusions : cette étude confirme l'intérêt de l'analyse toxicologique lors de la recherche des causes de décès en complément indispensable de l'autopsie.

\section{Intoxication mortelle par la gammabuty- rolactone (GBL), précurseur de l'acide gammahydroxybutyrique (GHB)}

M. DEVEAUX, F. BILLAULT, E. MÉDRANO, A. LE NOAN, L. NOEL, G. PÉPIN

Laboratoire TOXLAB, Paris, France

Objectif : décrire un cas d'intoxication mortelle, accidentelle, par ingestion de gammabutyrolactone (GBL), qui est un précurseur de l'acide gammahydroxybutyrique (GHB).

Description du cas : Des flacons de poppers et un litre de GBL conditionnée dans une bouteille d'eau minérale ont été achetés aux Pays-Bas pour une utilisation festive en France. La bouteille de GBL a été entreposée au réfrigérateur : un invité a bu par méprise une quantité non précisée de GBL, puis est allé se coucher. Il a été retrouvé décédé le lendemain matin. Des prélèvements de sang cardiaque, d'urine et de cheveux ont été effectués lors de l'autopsie. Du sang et de l'urine ont été prélevés sur trois autres participants. Différents liquides et verres ont été saisis et soumis également à l'analyse.

Méthodes : les fluides biologiques ont été analysés selon les procédures mises en œuvre en routine au laboratoire : l'éthanol par CPG-FID, les stupéfiants et les principaux médicaments par CLHP-BD et CPG-SM. Les cheveux étaient très courts et un seul segment a pu être analysé. La GBL et le GHB ont été dosés dans les liquides par CPGSM (colonne Factor Four, programmation de température : 65 à $315^{\circ} \mathrm{C}$ ) et CLHP-BD (colonne $\mathrm{C}_{18}$ Symmetry, phase mobile acétonitrile-tampon phosphate $\mathrm{pH} 3,8: 80$ 20). Le GHB a été dosé dans les humeurs par CPG-SM après dérivation par le BSTFA-TMCS, en utilisant le GHB- $\mathrm{D}_{6}$ comme étalon interne.

Résultats : deux flacons de poppers saisis contenaient bien du nitrite d'isobutyle dilué dans de l'isobutanol. Un autre contenait un mélange de nitrites d'alkyles. Le liquide contenu dans la bouteille d'eau minérale était bien de la GBL (88\%). Un des flacons de poppers et une autre bouteille contenait aussi de la GBL (74\%). Deux bouteilles de vin contenaient aussi des traces de GBL et de GHB. Les résultats des recherches effectuées dans les prélèvements des convives vivants \#1 à \#3 et de la victime \#4 sont les suivants :

\begin{tabular}{lcccc}
\multicolumn{1}{c}{ GHB } & $\# 1$ & $\# 2$ & $\# 3$ & $\# 4$ (décédé) \\
\hline Sang $(\mu \mathrm{g} / \mathrm{mL})$ & 28 & 8 & 12 & 1145 \\
Urine $(\mu \mathrm{g} / \mathrm{mL})$ & + & + & + & 5450 \\
Cheveux $(\mathrm{ng} / \mathrm{mg})$ & & & & 1,3
\end{tabular}

Accessoirement, d'autres stupéfiants (cannabinoïdes et benzoylecgonine) ont été aussi retrouvés dans le sang et les urines de tous les participants à cette soirée ; l'usage récréatif des poppers n'a pas pu être prouvé.

Conclusion : le GHB retrouvé dans les prélèvements biologiques de la victime \#4 provient donc de la GBL identifiée dans la bouteille (la GBL est le précurseur du GHB). La concentration sanguine de GHB dans le sang est du même ordre de grandeur que celles déjà décrites dans la littérature dans les cas de décès (338 à $3385 \mu \mathrm{g} / \mathrm{mL}$ ).

\section{Soumission chimique : autres molécules, mêmes conséquences !}

M. VILLAIN, V. CIRIMELE, G. SALQUEBRE, P. KINTZ

Laboratoire ChemTox, Division médico-légale, Illkirch, France

Objectif : démontrer que malgré la forte prévalence des benzodiazépines et hypnotiques dans les cas de soumission chimique, il est possible de trouver d'autres molécules à visée sédative impliquées dans ce type de situation. L'intérêt d'augmenter le panel des substances psychoactives criblées dans les cas de soumission chimique sera démontré au travers de 4 expertises judiciaires originales impliquant 2 neuroleptiques, 1 antihistaminique $\mathrm{H} 1$ et 1 antagoniste muscarinique.

Méthode : le laboratoire ChemTox a mis en place la stratégie analytique suivante : $1 /$ analyse du sang et des urines hydrolysées par HPLC-MS/MS après extraction par un mélange éther/dichlorométhane, à $\mathrm{pH} 8,4$, en présence de diazepam-d5 (étalon interne). 2/ analyse des cheveux segmentés et finement coupés par UPLCMS/MS, après décontamination, incubation dans $1 \mathrm{ml}$ de tampon phosphate $\mathrm{pH} 8,4$ et extraction. Cette approche permet de cribler les benzodiazepines inscrites à la Pharmacopée française, ainsi que les hypnotiques et les sédatifs suivants : promazine, alimémazine, cyamémazine, lévomepromazine, halopéridol, niaprazine, scopolamine, kétamine, diphénhydramine et 
doxylamine. Les limites de détection sont de l'ordre de 0,1 à $2 \mathrm{ng} / \mathrm{mL}$ dans le sang et les urines et 0,5 à $5 \mathrm{pg} / \mathrm{mg}$ dans les cheveux.

Résultats : Cas 1 : homme âgé, continuellement fatigué, en maison de retraite. Présence de promazine dans 3 segments de $2 \mathrm{~cm}$ aux concentrations de 9,2 et 6 $\mathrm{pg} / \mathrm{mg}$ alors que cette molécule ne fait pas partie de son traitement thérapeutique. Cas 2 : deux enfants placés chez leur belle-mère, régulièrement fatigués. Présence d'alimémazine dans l'ensemble des segments correspondant à la période de garde aux concentrations de 23 à $339 \mathrm{pg} / \mathrm{mg}$. Cas 3 : fillette de 9 ans violentée sous l'influence de diphénhydramine, retrouvée aux concentrations de 33 à 39 pg/mg, après analyse segmentaire des cheveux. Cas 4 : trois enfants chimiquement battus par de la scopolamine, dosée aux concentrations de 0,3 à $1,1 \mathrm{pg} / \mathrm{mg}$ sur une période d'au moins 6 mois.

Conclusion : dans l'ensemble de ces cas, l'administration de substances psychoactives a été confirmée ultérieurement. L'imagination des agresseurs n'ayant aucune limite, il est important de ne pas se limiter à la recherche des molécules les plus banales dans les affaires de soumission chimique.

\section{Évaluation de la pratique des prélève- ments à visée toxicologique à l'issue des autopsies au Maroc}

\section{H. WADIFI, N. HAMDOUNA, A. BELHOUSS, H. BENYAICH}

Service de Médecine Légale CHU Ibn Roch, Casablanca, Maroc

Dans la recherche des causes de la mort, et en particulier des morts suspectes, les analyses toxicologiques occupent une place importante en pratique médicolégale. Dans notre institut, les décès d'origine toxique constituent $7,6 \%$ des cas autopsiés.

Toutefois, la qualité de l'analyse toxicologique reste tributaire de la qualité des prélèvements, de leur conditionnement et conservation et des conditions d'acheminement au laboratoire.

Par ailleurs, l'analyse toxicologique ne peut se suffire à elle seule, mais repose sur une base associant anamnèse, constatations cliniques ou autopsiques en plus du savoir faire du laboratoire. D'où la nécessité d'une collaboration étroite entre le médecin légiste et l'analyste toxicologue.

Dans le but d'évaluer la pratique des prélèvements à visée toxicologique à l'issue des autopsies dans notre pays, nous avons entrepris une étude à travers un questionnaire que nous avons adressé aux responsables de 15 morgues municipales et hospitalières que nous avons interrogés sur la nature des échantillons prélevés, leur quantité, leur modalité de conservation et d'acheminement au laboratoire ainsi que sur leur relation avec les analystes toxicologues.

\section{Premier cas d'intoxication mortelle au dichlorvos en France}

J.C. ALVAREZ ${ }^{(1)}$, E. ABE ${ }^{(1)}$, C. DUVERNEUIL ${ }^{(1)}$, B. MATHIEU ${ }^{(1)}$, G. LORIN de la GRANDMAISON ${ }^{(2)}$

(1) Laboratoire de toxicologie ;

(2) Service de médecine légale, CHU R. Poincaré, Garches, France.

Objectif : un homme âgé de 54 ans est retrouvé décédé sur un terrain de football. Un flacon originaire du Maroc, contenant $100 \mathrm{ml}$ d'un liquide jaunâtre et portant les mentions "Denkavepon M 50", est découvert à proximité du corps. Une autopsie est pratiquée, montrant aucune lésion de violence et une congestion viscérale diffuse. Le contenu gastrique présente la même odeur que le liquide jaune. Une analyse toxicologique est demandée.

Méthodes : une recherche urinaire des stupéfiants (amphétamines, cannabis, cocaïne, opiacés, méthadone, buprénorphine, LSD) est réalisée par immunoanalyse (Abbott® et Microgenics ${ }^{\circledR}$ ) et confirmation en CPG-SM ou CL-SM/SM en cas de positivité. Une recherche non spécifique ("screening") après extraction liquide/liquide en milieu acide et basique et des recherches spécifiques des psychotropes (barbituriques, benzodiazépines, antidépresseurs et neuroleptiques) sont effectuées par CPG-SM, CLHP-BD et CLSM/SM. L'éthanol est dosé par CPG-FID. Une technique de dosage simple, rapide et reproductible du dichlorvos (DDVP) en CPG-SM a été mise au point et validée dans le sang total. $1 \mathrm{ml}$ d'échantillon est extrait par $4 \mathrm{ml}$ d'un mélange dichlorométhane/éther/hexane/alcool isoamylique $(150 / 250 / 100 / 2,5 ; \mathrm{v} / \mathrm{v} / \mathrm{v} / \mathrm{v})$ en milieu acide. Le DDVP-D ${ }_{6}$ est utilisé comme étalon interne. Le rendement d'extraction est de l'ordre de $100 \%$. La linéarité de la méthode est validée pour des concentrations comprises entre 0,5 et $10 \mu \mathrm{g} / \mathrm{mL}$. L'exactitude est comprise entre 92,7 et $100,2 \%$. Les $\mathrm{CV}$ intra et inter jours sont inférieurs à $15 \%$.

Résultats : l'analyse toxicologique a montré l'absence de toute substance médicamenteuse et stupéfiante. L'alcoolémie est négative. Seul est retrouvé au screening CPG/SM du dichlorvos (DDVP), organophosphoré utilisé comme insecticide. L'activité cholinestérasique du sang n'a pas été mesurée. Le DDVP est rapidement métabolisé au niveau hépatique, avec élimination des métabolites au niveau rénal, et présente une courte demi-vie chez l'homme (13,5 min). La technique développée a permis de mettre en évidence une concentration plus importante de DDVP dans le sang cardiaque $(4,4 \mu \mathrm{g} / \mathrm{mL})$ que dans le sang périphérique $(1,3 \mu \mathrm{g} / \mathrm{mL})$, différence probablement due à un relargage post-mortem depuis le myocarde. La forte concentration retrouvée dans le coeur semble en faveur de cette hypothèse $(1400 \mu \mathrm{g} / \mathrm{g})$. La plus faible concentration dans le poumon $(2,1 \mu \mathrm{g} / \mathrm{g})$ semble exclure une diffusion post-mortem depuis le contenu gastrique. La dif- 
fusion dans le rein est faible $(1,0 \mu \mathrm{g} / \mathrm{g})$, le DDVP dans le foie est indétectable, probablement dû à la présence des estérases hépatiques qui hydrolysent rapidement le DDVP. La concentration urinaire est de $1,3 \mu \mathrm{g} / \mathrm{mL}$. La concentration de DDVP dans le liquide gastrique, qui dégage une forte odeur aromatique d'ail, est de 253 $\mathrm{mg} / \mathrm{ml}$. La bouteille de "Denkavepon M50" contient du DDVP à la concentration de $550 \mathrm{mg} / \mathrm{ml}$. Il manque 150 $\mathrm{ml}$ dans la bouteille, correspondant à une dose présumée ingérée de $82 \mathrm{~g}$, soit environ $1 \mathrm{~g} / \mathrm{kg}$. La DL50 chez l'homme est inconnue, celle de la souris, du rat, du chien et du cochon s'établissant entre 50 et $150 \mathrm{mg} / \mathrm{kg}$.

Conclusion : la forte odeur observée au niveau gastrique lors de l'autopsie correspondait à la présence de DDVP, insecticide organophosphoré présentant une odeur aromatique caractéristique. Il s'agit du premier cas français d'intoxication mortelle au DDVP, pour lequel la distribution tissulaire est présentée, montrant un fort tropisme cardiaque de cette molécule, et confirmant sa forte toxicité.

\section{Toxicologie analytique}

\section{Exploration biologique de l'usage de cannabis : pourquoi et comment ?}

\section{P. MURA, B. BRUNET, Y. PAPET}

Service de Toxicologie et Pharmacocinétique, CHU, Poitiers, France

Objectif : la consommation de cannabis est en augmentation constante depuis plus d'une décennie dans de très nombreux pays. Compte tenu de ses effets délétères sur les fonctions cognitives et motrices, il est de plus en plus fréquemment impliqué dans les accidents de la voie publique, les accidents du travail, et plus généralement dans nombre de crimes et délits. Il n'est donc pas surprenant que l'exploration biologique des cannabinoïdes soit de plus en plus souvent confiée aux toxicologues analystes. L'objectif de ce travail est de répondre aux questions suivantes : (i) selon le contexte, quels sont les milieux biologiques les plus appropriés ? (ii) quelle(s) méthodologie(s) analytique(s) utiliser ? Comment interpréter les résultats ?

Méthodes : les réponses à ces questions sont apportées par les résultats des travaux effectués par la commission « drogues et conduite automobile » de la Société Française de Toxicologie Analytique ainsi que par ceux d'études récemment réalisées dans notre laboratoire.

Résultats : selon les études épidémiologiques récentes, le sur-risque d'accident (odds-ratio) de la circulation lié à un usage récent de cannabis est de 2 à 3 . Une étude française ayant portée sur 2003 conducteurs de moins de 30 ans décédés dans un accident de la circulation a montré que $39,6 \%$ d'entre eux avaient consommé du cannabis et que $28,9 \%$ en avaient consommé dans les heures précédentes. L'usage de cannabis constitue éga- lement une menace croissante de risque d'accidents du travail. Le nombre de délits et crimes (homicides volontaires, soumission chimique) associés à la présence de THC dans l'organisme des auteurs est aussi en augmentation. Pour mettre en évidence la responsabilité du THC dans les contextes précités, l'analyse sanguine est la plus appropriée. En effet, une étude rétrospective et dont les résultats sont présentés ici en primeur, montre une très mauvaise corrélation entre les signes cliniques objectifs de l'imprégnation cannabique (mydriase, irritation des conjonctives oculaires, etc.) et la présence de THC dans le sang. Si l'urine demeure le fluide biologique le plus efficace pour détecter une conduite addictive, par exemple en milieu professionnel, le sang est incontournable dans la plupart des autres domaines. En post-mortem et en l'absence de fluides biologiques, le tissu cérébral cerveau se révèle être tout à fait approprié comme l'a montré une étude récemment publiée. En ce qui concerne le dosage sanguin des cannabinoïdes, la méthodologie de référence est la chromatographie gazeuse couplée à la spectrométrie de masse. Si cette méthodologie permet de caractériser formellement le THC et ses métabolites et de les doser avec une excellente fiabilité, elle demeure longue et coûteuse. Nous avons étudié dans notre laboratoire la fiabilité d'un nouveau test immunochimique Elisa proposé pour rechercher les cannabinoïdes dans le sang total. L'analyse de 107 échantillons sanguins provenant de conducteurs impliqués dans un accident de la voie publique a révélé l'absence totale de faux négatifs ou de faux positifs. Cette méthodologie est donc particulièrement adaptée pour un dépistage rapide, préalablement à la confirmation des cas positifs par GC-MS. L'interprétation des résultats est toujours en débat mais de nombreux travaux permettent de conclure que la seule présence des principes actifs dans le sang indique que le sujet était sous influence au moment du prélèvement.

Conclusion : le toxicologue analyste dispose aujourd'hui de technologies tout à fait adaptées pour répondre à la demande croissante liée à ce nouveau fléau de santé publique.

\section{Confirmation rapide de la cocaïne, de ses métabolites et de produits apparentés dans les urines par CLHP-SM/SM : importance des standards internes deu- térés}

T. GOUGNARD, B. LAMNAOUAR,

\section{NEVE, J.M. MINON}

Laboratoire de Toxicologie, Service de Biologie Clinique, C.H.R. de la Citadelle, Liège, Belgique

Objectif : la technologie CLHP-SM-SM est devenue un outil performant au laboratoire de Toxicologie. A l'aide du protocole de validation de la SFTA (1), une méthode a été développée et caractérisée pour l'analy- 
se urinaire de la cocaïne, des métabolites principaux (benzoylecgonine, ecgonine méthylester) et de produits apparentés (anhydroecgonine méthylester, cocaéthylène). Parallèlement, la stabilité des courbes de calibration a été étudiée en fonction du choix des standards internes deutérés.

Méthode : la méthode fait appel à une CLHP-SM-SM triple quadrupole Waters/Micromass 2795 HT Quattro Micro et à une extraction conventionnelle sur phase solide "mixed-mode". Les standards internes sont la cocaïne-d3, la benzoylecgonine-d3 et l'ecgonine méthylester-d3. La phase mobile utilisée en mode gradient est composée d'acétate d'ammonium $10 \mathrm{mM}$ et d'acétonitrile. La colonne CLHP est une Zorbax* Eclipse XDB-C8 RR de $30 \mathrm{~mm}$ de long (2.1 $\mathrm{mm}$ d.i.), thermostatisée à $40^{\circ} \mathrm{C}$. En mode d'ionisation électrospray positif et acquisition MRM ("Multiple Reaction Monitoring"), un minimum de 2 ions-fils caractérise chaque substance. Une analyse dure 8 minutes.

Résultats : les rendements d'extraction oscillent entre 30 et $70 \%$. La suppression d'ionisation compte pour 30 à $50 \%$ de la réponse totale. Les limites de quantification varient de 2 à $16 \mathrm{ng} / \mathrm{ml}$. La gamme dynamique permet des déterminations jusqu'à $10.000 \mathrm{ng} / \mathrm{ml}$. Tous les coefficients de variation des substances analysées en dilution isotopique sont inférieurs à $10 \%$. Cependant, sur une période de 2 mois, pour la cocaéthylène et l'anhydroecgonine méthylester, l'absence d'équivalents deutérés a entraîné des variations inacceptables $(\mathrm{CV}>50 \%)$ et ce, malgré la parenté chimique et chromatographique.

Conclusion : le phénomène de suppression d'ionisation est un paramètre critique en mode électrospray. Il peut être la source d'un manque important de robustesse à toute méthode validée. La quantification systématique en dilution isotopique est un facteur de qualité pour les résultats obtenus au laboratoire de Toxicologie.

\section{Référence :}

1. Gruson A. et coll. Aide à la validation des méthodes en toxicologie et suivi therapeutique pharmacologique. Ann. Toxicol. Anal. 2005; vol. XVII, n³ ${ }^{\circ}$, suppl. 1

\section{Caractérisation d'atractylosides du chardon à glu par LC-MS. Application au cas d'une intoxication collective chez 6 enfants}

H. STAMBOULI, M. A. BELLIMAM

Laboratoire de Recherches et d'Analyses Techniques et Scientifiques, Rabat, Maroc

Objectif : mise au point d'une méthode de caractérisation d'atractylosides du chardon à glu par LC/MS et son application au cas d'une intoxication mortelle collective.

Méthode : la toxicité du chardon à glu est attribuée à deux hétérosides de la famille des diterpènes, l'atractyloside ATR (atractyline) et le carboxyatractyloside CATR (gummiférine), des poisons mitochondriaux inhibant la phosphorylation oxydative (cycle de Krebs) et s'opposant à la formation d'ATP à partir de l'ADP (1). La caractérisation des deux principes actifs de la plante (ATR et CATR) se fait souvent par des méthodes peu spécifiques : tests colorimétriques (réactifs de Marquis, Froedhe) et immunologiques. L'analyse chromatographique (CCM, HPLC, LC-MS) est peu développée et les données de la littérature ne fournissent pas assez de précisions quant aux conditions d'identification des hétérosides incriminés. Le présent travail propose la caractérisation en LC-MS des principaux atractylosides présents dans l'extrait éthanolique des rhizomes de la plante, puis l'application à l'analyse des fluides biologiques de victimes d'une intoxication collective. L'analyse par LC-MS est conduite en mode ESI négatif, sur une colonne Agilent C18 $(5 \mu \mathrm{m} ; 4,6 \times 250$ $\mathrm{mm}$ ), avec une phase mobile composée de tampon formiate d'ammonium $2 \mathrm{mM}$, pH 3 (A) et d'acétonitrile (B), en mode gradient linéaire (A : 80\% - B : 20\% 1 min ; A : $10 \%$ - B : $90 \% 20$ min ; A 10\% - B : $90 \% 5$ $\min$ ) avec un débit de $1 \mathrm{~mL} / \mathrm{min}$ et un split à la sortie de la colonne. La tension du capillaire est fixée à $-3 \mathrm{kV}$ et celle de la source à $-25 \mathrm{~V}$ ou à $-60 \mathrm{~V}$.

Résultats : l'ATR est caractérisé par un pic chromatographique au temps de rétention 5,50 min et par le spectre de masse de sa structure diprotonée, dominé par l'ion $725[\mathrm{M}-\mathrm{H}]^{-}$et son fils $362[\mathrm{M}-2 \mathrm{H}]^{2}-/ 2$. Ce résultat est obtenu avec la tension du cône $-25 \mathrm{~V}$. A -60 V, l'ion $[\mathrm{M}-\mathrm{H}]^{-}$perd $-\mathrm{SO}_{3}$ et conduit à la formation de l'ion fils $645\left[\mathrm{M}-\mathrm{H}-\mathrm{SO}_{3}\right]-$. Le troisième ion 481 s'explique par le départ simultané de $\left[-\mathrm{H},-2 \mathrm{SO}_{3},-\right.$ isovaryl]. Par ailleurs, 2 isomères d'atractylosides monodésulfatés [M 646] ont également été mis en évidence, respectivement à $8,27 \mathrm{~min}$ et $8,96 \mathrm{~min}$. Leurs spectres de masse sont semblables, se caractérisant tous deux par l'ion $645[\mathrm{M}-\mathrm{H}]$. Aucune trace du CATR n'a été détectée dans cet échantillon. Cette absence s'explique par la transformation du CATR en ATR par simple décarboxylation lorsque le matériel n'est pas frais et conservé longtemps au sec. En revanche, le travail sur une plante fraîche a permis de retrouver le pic chromatographique du CATR à 3,90 min et un spectre de masse obtenu à $-25 \mathrm{~V}$ caractérisé par l'ion $769[\mathrm{M}-\mathrm{H}]]^{-}$et son fils $384[\mathrm{M}-2 \mathrm{H}]^{2}-/ 2$. A la tension de $-60 \mathrm{~V}$, l'ion $[\mathrm{M}-\mathrm{H}]-$ génère l'ion 689 par la perte de -SO3-.

Application : cette méthode a été appliquée au cas d'une intoxication collective au chardon à glu de six enfants ( 3 filles et 3 garçons) d'une même famille, âgés de 5 à 12 ans, habitant en milieu rural. Parmi ces victimes une fille et un garçon âgés respectivement de 6 et de 10 ans ont trouvé la mort. Les prélèvements de sang, d'urine et de contenus gastriques des enfants intoxiqués sont traités à l'acétone pour précipiter les protéines, lavés au dichlorométhane, concentrés et leurs résidus sont repris par $100 \mu \mathrm{L}$ d'un mélange acétonitri- 
le/eau (50/50) pour injection. L'analyse dans les conditions décrites précédemment en LC-MS des extraits biologiques a conduit à la mise en évidence des traces d'ATR confirmant ainsi la consommation de la plante incriminée par les victimes.

Conclusion : la méthode de caractérisation d'atractylosides proposée, est inspirée des données de la littérature (2), mais elle fournit davantage de précisions sur les conditions d'analyse et d'identification des produits en LC/MS par leurs temps de rétention et spectres de masse. Elle a été ensuite appliquée au cas d'une intoxication infantile collective au chardon à glu.

\section{Références :}

1. Daniele C. et coll. J. Ethnopharmacology. 2005 ; $97: 175-81$.

2. Chèze M. et coll. Ann. Toxicol. Anal. 2000 ; 12(4) : 307-14.

\section{Caractérisation de l'éthylisme chronique (éthylglucuronide) dans les cheveux par HPLC-ESI-MS/MS}

\section{CIRIMELE, M. VILLAIN, G. SALQUEBRE, P. KINTZ}

Laboratoire ChemTox, Division médico-légale, Illkirch, France.

Objectifs : développer et valider une méthode de dosage de l'éthylglucuronide dans les cheveux pour la mise en évidence d'un éthylisme chronique.

Méthodes : après décontamination de la mèche de cheveux (deux bains successifs de dichlorométhane) et homogénéisation de l'échantillon (cheveux coupés en segments $<1 \mathrm{~mm}$ à l'aide de ciseaux), $100 \mathrm{mg}$ de la poudre ainsi obtenue sont incubés dans $1 \mathrm{ml}$ d'un mélange eau / méthanol (7:1, v/v) en présence de l'analogue pentadeutéré (25 ng d'éthylglucuronided5). Après $2 \mathrm{~h}$ de bain ultrasons, le milieu est centrifugé (15 min à 3000 RPM) puis la fraction limpide prélevée et évaporée. Après redissolution de l'extrait sec dans $50 \mu \mathrm{l}$ de phase mobile, $10 \mu \mathrm{l}$ sont injectés dans un système HPLC-ESI/MSMS. La séparation chromatographique est réalisée sur colonne X-Terra MS (3,5 $\mu \mathrm{m}, 2,1 \times 100 \mathrm{~mm}$ ) à l'aide d'une phase mobile composée d'acétonitrile / $0,1 \%$ acide formique en mode isocratique (5\%-95\%) et à un débit de $0,2 \mathrm{ml} / \mathrm{min}$. La détection est obtenue sur un Quattro Micro de chez Waters utilisant une interface de type electrospray fonctionnant en mode négatif (capillaire $3 \mathrm{kV}$, température de source $120^{\circ} \mathrm{C}$, débit de l'azote servant à la desolvatation $550 \mathrm{~L} / \mathrm{h})$. L'acquisition est réalisée en mode MRM (Multiple Reaction Monitoring) après optimisation des tensions de cône (22V) et de collision $(15 \mathrm{~V})$ propre à chacune d'entre-elles (éthylglucuronide : 221,1>74,8;221,1>84,8 et éthylglucuronide-d5 : $226,1>74,8 ; 226,1>84,8)$.

Résultats : la méthode analytique développée pour le dosage de l'éthylglucuronide dans les cheveux a été validée en terme de linéarité (20 à $500 \mathrm{pg} / \mathrm{mg}$, $\left.\mathrm{R}^{2}=0,9989\right)$, de limite de quantification $(20 \mathrm{pg} / \mathrm{mg})$ et de répétabilité intra- et inter-jour $(13,5$ et $18,4 \%$, respectivement à la concentration finale de $100 \mathrm{pg} / \mathrm{mg}$ ). L'application de la méthode validée à 61 échantillons issus de notre activité médico-légale a permis d'identifier 10 échantillons de cheveux positifs pour l'éthylglucuronide avec des concentrations comprises entre 30 et $953 \mathrm{pg} / \mathrm{mg}$.

Conclusion : une méthode analytique pour le dosage de l'éthylglucuronide dans les cheveux par HPLCESI/MSMS a été mise en place et validée. Elle est simple, rapide et permet de d'identifier des consommateurs réguliers de fortes quantités d'alcool. Les concentrations en éthylglucuronide généralement retrouvées dans les cheveux sont en moyenne très inférieures à celles retrouvées par exemple pour les opiacés, la cocaïne ou les amphétamines, c'est la raison pour laquelle un couplage spécifique et sensible comme l'HPLC-MS/MS est incontournable pour cette petite molécule polaire non volatile. Les résultats obtenus lors de cette étude se sont révélés être en accord avec ceux des études précédemment publiées et correspondent aux valeurs retrouvées chez les consommateurs réguliers d'alcool.

\section{Intoxications au carbofuran : mise au point analytique}

\section{A. EL BOURI, A. STAMBOULI, T. BOUAYOUN,} M.A. BELLIMAM, C. EL OUADOUDI

Laboratoire de Recherches et d'Analyses Techniques et Scientifiques, Rabat, Maroc

Objectif : l'étude propose une optimisation des conditions expérimentales d'analyse du pesticide carbofuran par GC/MS et la comparaison de cette méthode à la HPLC-DAD.

Méthode : la mise au point d'une méthode de caractérisation de carbofuran au laboratoire a été motivée par une série de dix affaires de vols perpétrés entre octobre 2003 et novembre 2005 dans des fermes privées, avec à chaque fois empoisonnement de chiens de garde à l'herbicide carbofuran. Dans les différents cas, ce carbamate a été détecté dans les fluides biologiques prélevés sur les chiens ou au sein des appâts consommés, par la technique GC/MS. Cette dernière qui présente l'inconvénient de dégrader le carbofuran, substance thermiquement instable (1), est pourtant préconisée au même titre que la HPLC avec détection fluorimétrique. Leurs limites de détection sont respectivement de 0,06 $\mu \mathrm{g} / \mathrm{L}$ (2) et $0,9 \mu \mathrm{g} / \mathrm{L}(3)$. Le présent travail consiste à optimiser les conditions expérimentales d'analyse du carbofuran par GC/MS afin de réduire les effets de sa dégradation thermique et à comparer cette méthode à la HPLC-DAD.

Résultats : en GC/MS, la décomposition du carbofuran 
en son dérivé phénolique, le 2,3-dihydro-2,2-dimethyl7-benzofuranol, sous l'effet de la température de l'injecteur $\left(270^{\circ} \mathrm{C}\right)$ est démontrée. Les deux pics chromatographiques sont enregistrés respectivement à 10,768 min et 7,414 min avec des spectres de masse caractéristiques des structures chimiques concernées (ions parents respectifs 221 et 164 uma). Un abaissement de la température d'injection de $270^{\circ} \mathrm{C}$ à $200^{\circ} \mathrm{C}$ permet d'atténuer le degré de cette dégradation qui passe de $16 \%$ à $6 \%$. Une température d'injecteur ramenée à $150^{\circ} \mathrm{C}$ conduit à l'élimination quasi-totale de la décomposition et aboutit à un seuil de détection de l'ordre de $0,5 \mu \mathrm{g} / \mathrm{L}$. Quant à la technique HPLC-DAD, elle permet de s'affranchir du problème de dégradation thermique du carbofuran puisqu'un seul pic chromatographique est obtenu à 6,547 min, et dont le spectre UV est caractérisé par une faible absorbance à $275 \mathrm{~nm}$. La limite de détection du carbofuran par cette technique a été évaluée à $1 \mu \mathrm{g} / \mathrm{L}$.

Conclusion : en définitive, l'étude a montré que la décomposition thermique du carbofuran en son dérivé phénolique lors de l'analyse par GC/MS peut être nettement minimisée en travaillant à une température d'injection de $150^{\circ} \mathrm{C}$. Dans ces conditions, la GC/MS offre une meilleure sensibilité $(\mathrm{LOD}=0,5 \mu \mathrm{g} / \mathrm{L}$ ) que la HPLC-DAD (LOD = $1 \mu \mathrm{g} / \mathrm{L})$.

\section{Références :}

1. Fournier J. Chimie des pesticides, cultures et techniques, Nantes. 1988.

2. Centre d'expertise en analyse environnementale du Québec, Méthode d'Analyse MA. 403-Pest-3.1, édition 2004.

3. US Environmental Protection Agency. Health advisory-Carbofuran. Office of Drinking Water, 1985.

\section{Identification et dosage de l'atractyloside et du carboxyatractyloside par CLHP- $\mathrm{SM}^{2}$ et CLHP-SM${ }^{3}$ dans le chardon à glu (Atractylis gummifera L.)}

L. ROMEUF ${ }^{(1)}$, Y. CHERRAH ${ }^{(2)}$, S. AHID ${ }^{(2)}$, M. OLLAGNIER ${ }^{(3)}$, T. BASSET ${ }^{(3)}$, Y GAILLARD ${ }^{(1)}$

(1) Laboratoire d'Analyses Toxicologiques, La Voulte sur Rhône, France ;

(2) Laboratoire de Pharmacologie, Rabat, Maroc ;

(3) Laboratoire de Toxicologie, Saint Etienne, France.

Objectif : les principes toxiques du chardon à glu sont des hétérosides à génine diterpénique qui inhibent le transport des nucléotides phosphorylés ADP et ATP empêchant la phosphorylation oxydative. Très puissants, ces toxiques sont la cause de nombreux décès aussi bien chez le bétail que chez l'Homme. Outre le chardon à glu qui est largement et tristement connu dans les pays du Maghreb, les genres Xanthium (les lampourdes) très répandus dans les deux Amériques et en Chine, Callilepis laureola DC dans le sud de
l'Afrique représentent d'autres sources d'intoxications mortelles. Les auteurs sud-africains décrivent de nombreuses insuffisances de leur technique $(1,2)$. La méthode que nous présentons décrit le dosage et l'identification parfaitement fiables des toxiques dans le matériel végétal et sert de point de départ pour le développement du dosage dans les fluides biologiques.

Méthode : l'atractyloside (ATR) et le carboxyatractyloside (CATR) sont des molécules sulfatées et les conditions requises pour leur séparation chromatographique et leur parfaite ionisation pour la spectrométrie de masse sont très délicates à obtenir et à conserver stables. La séparation est obtenue sur une colonne phényl X-terra ${ }^{\circledR}$ (Waters) de $150 \times 2,1 \mathrm{~mm}(3,5 \mu \mathrm{m}$ particules) au moyen d'un gradient ternaire composé de méthanol, d'acétonitrile et de tampon formate d'ammonium $2 \mathrm{mM}$ à $\mathrm{pH}=4,5$ tandis que la détection fait intervenir la spectrométrie de masse tandem et cube. L'ionisation est obtenue au moyen d'une source électrospray avec une tension inférieure à $3500 \mathrm{~V}$ en mode négatif. L'oxazépam D5 est utilisé comme étalon interne (EI) à défaut d'une structure chimique commercialement disponible qui soit comparable aux analytes. Le CATR montre une forte instabilité dans les solvants protéiques comme le méthanol.

Résultats : les temps de rétention du CATR, ATR et EI sont respectivement de 9,6;11,0 et 14,8 min. En SM cube, L'ion fils majoritaire du CATR est le $\mathrm{m} / \mathrm{z}=645$, fils $\mathrm{du} \mathrm{m} / \mathrm{z}=689$ fils $\mathrm{du} \mathrm{m} / \mathrm{z}=769$ tandis que pour l'ATR l'ion fils majoritaire est le $\mathrm{m} / \mathrm{z}=543$, fils du $\mathrm{m} / \mathrm{z}$ $=645$, fils $\mathrm{du} \mathrm{m} / \mathrm{z}=725$. La racine du chardon à glu collectée au Maroc a été séchée puis réduite en poudre. La concentration en ATR est de $0,2 \%(n=6)$ et celle en CATR est $5,7 \%(n=6)$ exprimé en poids de matière sèche.

Conclusions : ce rapport est le premier donnant une mesure de concentration en principe actif dans le chardon à glu Atractylis gummifera L. La connaissance de la chimie de l'ATR et du CATR que nous avons acquise est mise à profit pour développer une méthode d'extraction en phase solide sur support cationique faible autorisant enfin l'accès aux fluides biologiques.

\section{Références :}

1. Steenkamp P.A. et coll. Identification of atractyloside by LC-ESI-MS in alleged herbal poisonings. Forensic Sci Int. 2006 ; 163 : 81-92.

2. Laurens J.B. et coll. Gas chromatographic-mass spectrometric confirmation of atractyloside in a patient poisoned with Callilepis laureola. J Chomatogr B Biomed Sci Appl. 2001; 765 : 127-133. 


\section{Dépistage toxicologique ciblé et non ciblé par LC-MS. Liens entre l'évolution des techniques et les pratiques en toxicologie analytique}

K. AMOURA

Waters ${ }^{\circledR}$, Saint-Quentin-en-Yvelines, France.

La technique LC-MS est une méthode de confirmation de référence. La majorité des laboratoires de toxicologie, oeuvrant dans le domaine légal, sont aujourd'hui convaincus par cette technologie et tente d'améliorer son utilité en développant des méthodes de dépistage ciblées.

Les laboratoires de toxicologie clinique sont à la recherche, à la fois, d'un outil de dépistage large avec automatisation des identifications, et d'un outil de confirmation et de dosage.

Tous souhaitent avoir accès à des solutions qui limitent les étapes de préparation des échantillons et qui diminuent le plus possible les retraitements et les interprétations de résultats.

Waters ${ }^{\circledR}$ propose aujourd'hui plusieurs plateformes analytiques. Chacune d'entre-elles permet de répondre totalement ou partiellement aux demandes des toxicologistes. L'exposé développera ces différentes approches et précisera les bénéfices et les limites de chacune.

\section{Résultats de toxicologie et prise de décision}

\section{Cheveu et toxicologie judiciaire. Que peut-on en attendre en 2006 ?}

P. KINTZ, M. VILLAIN, G. SALQUEBRE, V. CIRIMELE

Laboratoire ChemTox, Division médico-légale, Illkirch

Objectif : en France, dans les années 90, l'utilisation des phanères en toxicologie était vue comme une curiosité, parfois avec un grand scepticisme, compte tenu des faibles quantités à identifier, de l'ordre du ng. De nos jours, ce prélèvement alternatif est totalement accepté par la Justice et constitue la base de nombreuses expertises impliquant la soumission chimique, le dopage, l'empoisonnement criminel ou les recherches des causes de la mort. Si l'aspect analytique est de mieux en mieux maîtrisé, en particulier par la mise en place de contrôles de qualité, la difficulté majeure dans ce domaine reste l'interprétation.

Méthode : la Society of Hair Testing a publié des recommandations (1) pour les stupéfiants. Après un dépistage par RIA ou ELISA, la GC/MS s'impose. Dans le cadre du dopage et de la soumission chimique, puisque les concentrations attendues sont de l'ordre du pg, la spectrométrie de masse en tandem est indispensable.
Résultats : dans un premier temps, la stratégie analytique du laboratoire ChemTox sera présentée. Pour les stupéfiants, elle fait appel à une incubation méthanolique, un criblage par ELISA IDS OneStep et une confirmation directe sur l'extrait par GC/MS. Pour les autres substances, après une incubation dans un tampon phosphate $\mathrm{pH} 8,4$ et une extraction simple ether/dichlorométhane, les couplages GC/MS et UPLC/MS-MS sont mis en œuvre.

Parmi les biais liés à l'analyse de cheveux, les 4 paramètres suivants présentent une importance majeure dans l'interprétation des résultats : couleur des cheveux, vitesse de pousse, influence des traitements cosmétiques et influence du milieu de conservation.

La mélanine, site de liaison des xénobiotiques est le pigment responsable de la couleur des cheveux. Sa concentration et ses iso-formes conduisent à des concentrations capillaires variables après une dose équivalente pour les molécules basiques mais pas les neutres.

Les traitements cosmétiques, en particulier les colorations peuvent conduire à une diminution de concentrations ou à une migration anarchique du spot d'exposition. La conservation au réfrigérateur des cheveux conduit à une perte de 30 à $60 \%$ du contenu en xénobiotiques (problème des kits d'autopsie conservés à $+4^{\circ} \mathrm{C}$ ).

Les cheveux (ayant baigné dans le jus de putréfaction) d'un cadavre exhumé, décédé d'une intoxication massive à la cyamémazine (alors que non traité par du Tercian $\left.{ }^{\circledR}\right)$ se sont trouvés contaminés par le neuroleptique avec des concentrations uniformes le long de la mèche (par segmentation), sans qu'il soit possible éliminer cette contamination. Il en est de même chez les sujets décédés dans un contexte de toxicomanie à l'ecstasy, lorsque la phase agonique induit une hypersudation qui favorise l'incorporation externe de MDMA.

Finalement, chez les noyés, l'eau douce ne semble avoir qu'une action modeste sur les concentrations alors que l'eau de mer conduit invariablement à une extraction massive des xénobiotiques.

Conclusion : il apparaît donc nécessaire, dans tout rendu de résultat d'analyse de cheveux, d'indiquer dans quelles circonstances ils ont été prélevés, leur couleur, la longueur du segment analysé et sa masse (influence sur la limite de quantification).

\section{Référence :}

1. Society of Hair Testing. Recommandations pour les analyses de cheveux dans les expertises médicolégales. Ann. Toxicol. Anal. 2005 ; 17 : 217-9. 


\section{Soumission chimique: quand le cheveu devient indispensable. A propos de trois cas}

\author{
V. DUMESTRE-TOULET ${ }^{(1)}$, P. KINTZ ${ }^{(2)}$
}

(1) Laboratoire ToxGen, Bordeaux ;

(2) Laboratoire ChemTox, Illkirch, France.

Objectif : nous présentons ici plusieurs cas de discrimination entre une exposition unique et un usage répété de xénobiotiques psychotropes par l'analyse séquentielle des cheveux de sujets soumis chimiquement par un agresseur ou un parent.

Description des cas : $\underline{\text { Cas } 1}$ : Une jeune femme de 21 ans est victime d'un viol sur une aire d'autoroute après avoir absorbé un comprimé blanc. Cas 2 : Une enfant de 5 mois décède à l'hôpital après absorption de méthadone. Cas 3 : Deux garçons adolescents sont abusés sexuellement après avoir sniffé une poudre blanche qu'ils croyaient être de la cocaïne. Dans tous les cas il $\mathrm{y}$ a eu judiciarisation et des expertises toxicologiques ont été requises avec analyses de fluides biologiques et de cheveux afin de mettre en évidence les éventuelles substances absorbées et la durée d'exposition.

Méthodes : des prélèvements de sang, d'urines, parfois de bile et de viscères ainsi que de cheveux ont été analysés selon les procédures habituelles du laboratoire. L'alcool est mesuré par CPG/IF, les stupéfiants et les médicaments psychoactifs sont recherchés par technique ELISA sur microplaques, CLHP/BD et CPG/SM dans les fluides biologiques. Les recherches de médicaments psychotropes ont été effectuées dans des segments de cheveux des victimes par CL-SM/SM ou CPG-SM/SM après incubation dans un tampon et extraction liquide/liquide en présence d'étalons internes.

Résultats : Cas 1 : Le bromazépam $\left(\right.$ Lexomil $\left.^{\circledR}\right)$ a été identifié dans le sang à une concentration de $151 \mathrm{ng} / \mathrm{mL}$, et dans les cheveux à des concentrations respectivement de 7,5 et $0,9 \mathrm{pg} / \mathrm{mg}$ dans les segments $0-2 \mathrm{~cm}$ et $2-4 \mathrm{~cm}$. Cas 2 : La méthadone est identifiée dans le sang et les urines avant le décès à des concentrations respectivement de 142 et $466 \mathrm{ng} / \mathrm{mL}$ et dans plusieurs segments de cheveux à des concentrations de $1,1,6$ et $2,3 \mathrm{ng} / \mathrm{mg}$. Cas 3 : Le 7 amino clonazépam (Rivotril $^{\circledR}$ ) est identifié dans le sang, les urines et les cheveux aux concentrations respectives de $7 \mathrm{ng} / \mathrm{mL}$, $58 \mathrm{ng} / \mathrm{mL}, 141 \mathrm{pg} / \mathrm{mg}$ pour l'un des garçons et de $10 \mathrm{ng} / \mathrm{mL}, 233 \mathrm{ng} / \mathrm{mL}, 336 \mathrm{pg} / \mathrm{mg}$ pour l'autre.

Conclusion : plusieurs cas de soumission chimique avec du bromazépam et du clonazépam sont décrits dans la littérature avec des concentrations très faibles mesurées dans les cheveux. Des cas d'intoxications d'enfants avec de la méthadone sont également décrits. Dans les cas présentés, les analyses de cheveux étaient en faveur d'une exposition unique (cas 1 et 3) ou bien chronique (cas 2) infirmant ou confirmant les déclarations des victimes et/ou agresseurs présumés et éclairant ainsi l'enquête de police. Les auteurs insistent sur l'importance des analyses de cheveux dans les cas de soumission chimique nécessitant des techniques ultrasensibles, non disponibles dans tous les laboratoires de toxicologie, mais accessibles par le biais d'une dualité d'experts.

\section{Intoxication au flécaïnide : présentation d'un cas et revue de la littérature}

\section{A. SBIHI, A. KETTANI, A. MOSADIK,} Z.H. BELKHADIR, M. FAROUDY

Service de la Réanimation des Urgences Chirurgicales, CHU Ibn Sina, Rabat, Maroc

Objectif : faire le point, à travers une observation et une revue de la littérature, sur la physiopathologie, la présentation clinique, les facteurs de gravité et la prise en charge thérapeutique d'une intoxication peu connue et de mauvais pronostic.

Méthodes : observation personnelle et revue systématique de la littérature (Pubmed®, 1980-2006) (1-3).

Observation : patiente de 28 ans, admise pour ingestion 2 heures plus tôt, de $2 \mathrm{~g}$ flécainide appartenant à son mari dans une tentative d'autolyse. Parfaitement stable à l'admission, elle a présenté après $2 \mathrm{~h}$ un état de choc avec une brady arythmie à $35 \mathrm{bpm}$ faite de complexes QRS larges à type d'échappement ventriculaire. L'évolution était bonne après administration d'atropine et mise sous dopamine à dose alpha pendant $4 \mathrm{~h}$. L'ECG de contrôle était strictement normal, ainsi que l'échocardiographie réalisée le lendemain. Le flécaïnide a été retrouvé dans le liquide gastrique et le sang, mais les taux plasmatiques n'ont pas pu être obtenus.

Discussion et conclusion : les intoxications par le flécaïnide, antiarythmique de la classe 1c de VaughanWilliams, sont rares mais graves, notamment à cause de la dépression myocardique et des troubles sévères du rythme et de conduction cardiaque. La prise en charge, d'efficacité aléatoire, est essentiellement symptomatique : lavage gastrique, oxygénothérapie, atropine si bradycardie, catécholamines si défaillance circulatoire, sels de sodium molaire si troubles de conduction sévères. La rareté de cette intoxication explique la méconnaissance fréquente du traitement adapté et l'absence d'administration de sels de sodium chez notre patiente. Le pronostic est lié principalement à la dose et au terrain. Le lavage gastrique précoce, le jeune âge et l'absence d'antécédents cardiaques peuvent expliquer la bonne évolution chez notre patiente.

\section{Références :}

1. André P. et coll. A propos d'un cas d'intoxication par la flécaïne. Urgences. $1997 ; 16$ : 205-7.

2. Winkelmann BR et coll. Life-threatning flecainide toxicity : A pharmacodynamic approach. Ann Intern Med. 1987 ; 106(6) : 807-14.

3. Koppel C. et coll. Clinical course and outcome in class Ic anti-arrhythmic overdose. Clin Toxicol. 1990 ; $28: 433-44$. 


\section{L'évolution de la toxicologie analytique permet-elle toujours d'élucider les causes de la mort?}

\author{
B. BRUNET ${ }^{(1)}$, Y. PAPET ${ }^{(1)}, \quad$ P. KINTZ $^{(2)}$, \\ M. SAPANET ${ }^{(3)}$, P. MURA ${ }^{(1)}$ \\ (1) Service de Toxicologie et Pharmacocinétique, \\ CHU, Poitiers ;
}

(2) Laboratoire ChemTox, Division médico-légale, Illkirch ;

(3) Service de Médecine Légale, CHU, Poitiers, France.

Objectif : l'objectif est de montrer l'évolution des différentes techniques analytiques au cours des trente dernières années et d'en présenter les avantages et les limites, illustrées par deux cas.

Méthodes : dans les deux cas présentés, des techniques analytiques parmi les plus performantes à ce jour ont été utilisées : HPLC-DAD, HS-GC/MS, GC-MS/MS.

Résultats : $\underline{\text { Cas } n^{\circ} 1}$ : un homme de 60 ans, psychotique chronique, est admis aux urgences hospitalières pour un syndrome fébrile et une pneumopathie. Une oxygénation au masque et une antibiothérapie sont mises en place. Le lendemain, le patient est retrouvé en arrêt respiratoire avec une mydriase bilatérale et une turgescence jugulaire. La tubulure de la perfusion est retrouvée branchée sur le tuyau du masque à oxygène avec un débit de $10 \mathrm{~L} / \mathrm{min}$. Une autopsie est pratiquée et une expertise toxicologique est demandée par la Justice pour recherche des causes de la mort. L'analyse des échantillons biologiques a mis en évidence une alcoolémie nulle, une carboxyhémoglobine à $0,6 \%$, des cyanures inférieurs à $50 \mathrm{ng} / \mathrm{mL}$ et un GHB à 2,6 $\mathrm{mg} / \mathrm{mL}$. L'analyse des gaz par HS-GC/MS n'a pas permis de mettre en évidence une anomalie de composition. Cas $n^{\circ} 2$ : un homme de 17 ans est retrouvé sur les toilettes dans un réduit de $3,6 \mathrm{~m} 3$, avec 3 bombes de laque en aérosol vides. A l'autopsie sont notés un œdème aigu du poumon et une importante congestion viscérale. L'analyse toxicologique du sang révèle la présence de THC $(1,5 \mathrm{ng} / \mathrm{mL})$, de THC-COOH $(8,6$ $\mathrm{ng} / \mathrm{mL})$ et d'éthanol $(0,56 \mathrm{~g} / \mathrm{L})$. La recherche de substances volatiles est négative.

Conclusions : ces deux cas illustrent bien les limites de l'expertise toxicologique lorsque des fluides physiologiques gazeux sont en cause. Sans la connaissance des rapports d'autopsie et des données de l'anamnèse, le toxicologue analyste aurait été dans l'impossibilité de se prononcer sur les causes de la mort.

\section{Injection mortelle d'alfentanil (Rapifen ${ }^{\circledR}$ )} V. DUMESTRE-TOULET ${ }^{(1)}$, M. VILLAIN $^{(2)}$, P. KINTZ $^{(2)}$

(1) Laboratoire ToxGen, Bordeaux ;

(2) Laboratoire ChemTox, Illkirch, France.

Objectif : nous rapportons ici le cas d'une intoxication létale par injection d'alfentanil (RAPIFEN ${ }^{\circledR}$, ampoules de 1 et $5 \mathrm{mg}$ ), analgésique puissant apparenté chimique- ment au fentanyl et utilisé en anesthésie générale sous ventilation assistée et surveillance cardiorespiratoire.

Description du cas : un infirmier anesthésiste de 46 ans est retrouvé décédé dans les toilettes d'une clinique chirurgicale. Une seringue est plantée dans son bras, un garrot retrouvé à ses pieds. Il est soupçonné d'être toxicomane aux opioïdes anesthésiques car des ampoules disparaissent, brisées, depuis plusieurs mois dans le service. A l'autopsie, le médecin légiste constate une congestion viscérale intense, en particulier des poumons. Une expertise toxicologique est requise afin de mettre en évidence les éventuelles substances absorbées.

Matériels et méthodes : les différents prélèvements effectués par le médecin légiste (sang, urine, cheveux) ont été analysés selon les procédures habituelles du laboratoire. L'alcool est mesuré par CPG/IF, les stupéfiants et les principaux toxiques sont recherchés par CHLP/BD et CPG/SM. L'alfentanil a été identifié par $\mathrm{CPG} / \mathrm{SM}$ puis quantifié par $\mathrm{CPG} / \mathrm{SM} / \mathrm{SM}$ après extraction en milieu alcalin à $\mathrm{pH} 9,5$ par le mélange dichlorométhane/isopropanol/n heptane $(33: 17: 50, \mathrm{v} / \mathrm{v} / \mathrm{v})$ en présence de fentanyl d5 comme étalon interne.

Résultats et discussion : l'alfentanil a été identifié dans le sang et les urines à des concentrations respectives de 45,1 et $2,7 \mathrm{ng} / \mathrm{mL}$, ce qui indique un décès très rapide. Les analyses révèlent aussi une alcoolémie de $1,32 \mathrm{~g} / \mathrm{L}$. La recherche effectuée dans une mèche de cheveux de $3 \mathrm{~cm}$ met en évidence la présence d'alfentanil $(2 \mathrm{pg} / \mathrm{mg})$ et de fentanyl $(8 \mathrm{pg} / \mathrm{mg})$.

Les concentrations thérapeutiques habituelles après l'injection initiale de 8 à $50 \mu \mathrm{g} / \mathrm{kg}$ pour obtenir une analgésie chirurgicale sont associées à des concentrations plasmatiques de 200 à $1000 \mathrm{ng} / \mathrm{mL}$. Plusieurs cas de dépressions respiratoires sévères avec hypotension et coma sont décrits lors d'interventions chirurgicales malgré une ventilation assistée pour des concentrations sériques supérieures à $100-200 \mathrm{ng} / \mathrm{mL}$ (1). Alors que de nombreux cas sont relatés dans la littérature avec le fentanyl (2), il semble que la toxicomanie à l'alfentanil soit exceptionnelle (3).

Conclusion : bien que les concentrations mesurées soient thérapeutiques, les analyses réalisées dans le sang et les urines du sujet sont en faveur d'un décès par overdose d'alfentanil, en l'absence d'assistance respiratoire. La présence dans les cheveux indique une consommation chronique du produit, compatible avec une conduite addictive.

\section{Références :}

1) Mahla M.E. et coll. Delayed respiratory depression after alfentanil. Anesthesiology 1988; 69: 527-34.

2) Villain M. et coll. Toxicomanie d'un anesthésiste au fentanyl : la preuve formelle par analyse des cheveux en utilisant la CPG-SM/SM. Ann. Toxicol. Anal. 2001; 13(1): 49-53.

3. Kintz P. et coll. Conduites addictives en milieu hospitalier : particularités d'un service d'anesthésie. Ann. Toxicol. Anal. $2002 ; 14(1): 83-9$ 


\section{Thèmes libres}

\section{Validation d'une méthode analytique pour le dosage du méthanol dans le sang et les urines : application à deux cas d'intoxication}

\author{
M.A. BELLIMAM, A. STAMBOULI, T. BOUAYOUN,
} A. EL BOURI

Laboratoire de Recherches et d'Analyses Techniques et Scientifiques de la Gendarmerie Royale, Rabat

Objectifs : le méthanol est utilisé comme solvant organique, fluide d'essuie-glace de pare-brise, antigel d'essence, décapant, et aussi comme dénaturant des alcools à brûler. Les intoxications au méthanol décrites dans la littérature sont volontaires ou accidentelles, dues essentiellement au déconditionnement du produit ou à la consommation d'alcool frelaté.

Le méthanol est résorbé dans le tractus gastro-intestinal, son pic plasmatique est atteint au bout de 30 à 90 min (1). Il est principalement métabolisé au niveau du foie, par l'alcool déshydrogénase (ADH) et l'aldéhyde déshydrogénase (ALDH) en son métabolite toxique, l'acide formique (2). Ce travail présente l'analyse du méthanol par spectrométrie RMN et GC/MS puis décrit la validation d'une méthode de son dosage dans les urines et le sang par GC/MS. Dans cette étude, nous rapportons le cas d'une intoxication de deux hommes, âgés de 33 et 34 ans, retrouvés morts. De leurs vivants, ils s'adonnaient à l'alcool à brûler, en vente libre dans les épiceries.

Méthodes : le liquide resté dans la bouteille incriminée est analysé à la fois par spectrométrie $\mathrm{RMN}{ }^{1} \mathrm{H},{ }^{13} \mathrm{C}$ et par HS-GC/MS. Alors que son dosage dans les liquides biologiques est effectué par HS-GC/MS. A cet effet, $1 \mathrm{~mL}$ du liquide biologique est placé dans un flacon à fermeture hermétique, auquel sont rajoutés $1 \mathrm{~g}$ de sulfate d'ammonium et $50 \mu \mathrm{L}$ d'isopropanol $(50 \mathrm{~g} / \mathrm{L})$ utilisé comme standard interne. Le flacon est ensuite fermé, étuvé à $80^{\circ} \mathrm{C}$ pendant $15 \mathrm{~min}, 1 \mathrm{~mL}$ de l'espace de tête est injecté en GC/MS. La quantification du méthanol et de l'isopropanol se fait respectivement par reconstitution des ions 31 et 45 .

Résultats : les résultats d'analyses du liquide restant dans la bouteille, supposé consommé par les victimes, ont montré la présence exclusive du méthanol, attestant que ce produit correspond à de l'alcool à brûler frelaté. La droite d'étalonnage du méthanol est linéaire jusqu'à $20 \mathrm{~g} / \mathrm{L}$ avec un coefficient de corrélation de 0,995 . La $\mathrm{LD}$ et la LQ sont respectivement de 0,005 et $0,016 \mathrm{~g} / \mathrm{L}$ avec un CV de $16 \%$ pour l'urine et $11 \%$ pour le sang. La répétabilité $(\mathrm{n}=10)$ pour une solution de $3 \mathrm{~g} / \mathrm{L}$ a été obtenue avec un CV de $13 \%$ et la justesse a également été déterminée pour une valeur cible de $3 \mathrm{~g} / \mathrm{L}(\mathrm{n}=5)$, et les valeurs calculées rentrent dans la fourchette d'incertitude de $20 \%$. Cette méthode appliquée aux deux cas d'intoxication étudiés, a permis de déterminer les teneurs du méthanol dans le sang et les urines respectivement à $2,5 \mathrm{~g} / \mathrm{L}$ et $0,6 \mathrm{~g} / \mathrm{L}$ dans le premier cas et à $2,6 \mathrm{~g} / \mathrm{L}$ et $1,5 \mathrm{~g} / \mathrm{L}$ dans le second cas.

Conclusion : les teneurs élevées en méthanol dans le sang comparativement à celles déterminées dans les urines s'expliquent par la métabolisation du méthanol en acide formique. Il est à signaler, que l'acide formique a été mis en évidence qualitativement de façon plus importante dans les urines que dans le sang.

\section{Références :}

1. Ellenhorn M.J. et Barceloux D.G. Medical toxicology: diagnosis and treatment of human poisoning, Baltimore, Williams \& Wilkins; 1988.

2. Ellenhorn M.J. Ellenhom's medical toxicology: diagnosis and treatment of human poisoning, $2^{\text {nd }}$ ed., Baltimore, Williams \& Wilkins; 1997.

\section{Audit clinique des décès par l'envenima- tion scorpionique (ES) : Première expé- rience marocaine}

\section{SEMLALI $^{(1)}$, R. SOULAYMANI ${ }^{(1)}$, D. BACHEIKH ${ }^{(2)}$}

(1) Centre Anti Poison et de Pharmacovigilance du Maroc (CAPM), Rabat, Maroc ;

(2) Consultant en santé publique, spécialiste dans les aspects méthodologique de l'audit.

Introduction : dans le cadre de l'appui de la stratégie marocaine de lutte contre les piqûres et les envenimations scorpioniques (P.E.S) qui est axée sur l'amélioration de la prise en charge (PEC) des PES, une démarche d'audit clinique des décès par ES a été adoptée pour la première fois par le CAPM, puis testée et implantée au niveau de 2 hôpitaux pilotes HA et HB.

Objectifs : présenter les différentes phases réalisées pour l'implantation de l'audit clinique des décès par ES, les résultats des séances d'audit au niveau des 2 hôpitaux pilotes et aussi dégager la perception des personnes impliquées dans l'audit.

Matériels et méthodes : un système d'information spécifique a été mis en place au niveau de 2 hôpitaux pilotes. Il s'agit de la fiche d'hospitalisation résumant le cas clinique, la fiche de synthèse d'audit comportant un tableau d'évaluation de la conformité aux critères de la qualité de PEC avec un score de cotation de la validité de ces critères, l'identification des dysfonctionnements, les facteurs en cause et les propositions des solutions correctives. Tous les décès par ES de l'année 2005 pour les 2 hôpitaux ont été audités, notifiés sur ces supports et envoyées au CAPM pour analyse. Les données ont été analysées par Excel.

De plus, les acteurs impliqués dans l'audit ont répondu sur un questionnaire qui a été porté sur le cycle de l'audit, l'apport de l'audit et les barrières à sa réalisation.

Résultats : le résultat de l'analyse des données pour les 33 décès notifiés ( 20 de HA et 13 de HB), a montré que le profil des décédés est différent entre les 2 hôpitaux. 
Des dysfonctionnements relatifs à la mise en condition, à la conduite thérapeutique et au suivi des patients, représente chacun $23,5 \%$ des dysfonctionnements pour $\mathrm{HA}$, alors que $50 \%$ des dysfonctionnements sont dus au long délai de transfert pour HB. Le score de validité des critères de la qualité de la PEC a été pour HA et HB en moyenne de 3/4 et de 3,69/4 respectivement. Les causes de ces dysfonctionnements se résument dans le manque de certains médicaments dans $2 / 3$ des causes, l'incompétence professionnelle dans $1 / 3$ des causes pour $\mathrm{HA}$ et le manque de matériel dans $83,33 \%$ des causes pour HB. Le questionnaire a montré pour les 2 hôpitaux que le cycle de l'audit a été respecté dans ses normes mais on a noté une irrégularité dans la réalisation des séances à cause de la non disponibilité des membres impliqués. Comme apport d'audit on a noté un changement d'attitude du personnel avec une prise de conscience des problèmes et une augmentation de la vigilance, l'organisation des tâches, la standardisation du protocole thérapeutique, une formation locale a été initiée, la communication et la coordination entre les services ont été établies surtout pour HA. Néanmoins certaines contraintes ont été observées quant à l'adhésion au référentiel, le manque d'un bon climat de travail, démotivation et résistance de certaines personnes pour HA.

Conclusion : la pratique d'audit est une culture nouvelle dont on doit comprendre la philosophie, mais reste faisable. Néanmoins, pour réussir le processus d'audit, il faut un comité d'audit représentatif et qualifié, un encadrant de près, un suivi régulier avec un soutien administratif rigoureux et une supervision bien programmée pour évaluer les progrès apportés.

\section{Références :}

1. Soulaymani-Bencheikh R. et coll. Stratégie nationale de lutte contre les piqûres et les envenimations scorpioniques. Bull. Soc. Pathol. Exot. 2003; 96: 317-9.

2. Soulaymani-Bencheikh R. et coll. Situation épidémiologique des piqûres de scorpion au Maroc Cartes d'incidence, de létalité, de mortalité selon les régions (2001-2004)

http://www.monednat.org/article.php3?id_article=52

Utilisation des cheveux comme un indicateur de la bioaccumulation des éléments traces chez les enfants : Nouvelle approche d'une procédure de lavage

Y. BARKOUCH ${ }^{(1)}$, A. SEDKI ${ }^{(2)}$, J.C. AMIARD ${ }^{(3)}$, A. PINEAU ${ }^{(1)}$

(1) Laboratoire de Toxicologie et d'hygiène industrielle, CHU, Nantes, France ;

(2) Laboratoire d'Hydrobiologie, Ecotoxicologie et Assainissement, Marrakech, Maroc ;

(3) PPF Pôle mer et littoral, Nantes, France.

Objectif : en toxicologie, plusieurs indicateurs biolo- giques (sang, urines, ongles, lait...) sont utilisés pour indiquer le taux d'imprégnation de l'organisme humain par les éléments minéraux, indication qui souvent exige le recours au dosage sanguin. Cette matrice nécessite des conditions de prélèvement et de conservation très strictes. La vision actuelle tend vers l'utilisation d'un matériel biologique facile à récupérer et facile à conserver : les cheveux (1). Le dosage des métaux lourds dans les cheveux peut être associé à des erreurs. En réalité, le dosage doit tenir compte uniquement de la fraction intra-pilaire (endogène) $(1,2)$, d'où la grande nécessité de se débarrasser de la fraction de contamination extrapilaire (exogène) provenant du dépôt des poussières riches en métaux (1). L'objectif de cette étude est de comparer l'ampleur de l'action mobilisante de différentes procédures de lavage afin de choisir une méthode efficace permettant d'éliminer la totalité ou la majorité des éléments traces d'origine externe qui se sont déposés sur la tige pilaire, sans être agressive au point de toucher à l'intégrité de la matrice pilaire en mobilisant la fraction d'origine interne qui seule reflète l'imprégnation de l'organisme (1).

Méthode : la méthode utilisée consiste d'abord à simuler une contamination artificielle volontaire des cheveux dont nous suivons l'élimination par sept procédés de lavage. Le dosage des éléments traces métalliques ( $\mathrm{Al}, \mathrm{As}, \mathrm{Cd}, \mathrm{Cu}, \mathrm{Ni}, \mathrm{Pb}$, Se et $\mathrm{Zn}$ ) est effectué par spectroscopie d'absorption atomique sur les échantillons de cheveux digérés à l'acide nitrique $8 \mathrm{~N}$.

Résultats : 1/Étude de l'efficacité de lavage : - tous les produits utilisés contribuent à une élimination (totale ou partielle) de la fraction exogène. L'efficacité de lavage dépend du produit utilisé, de sa concentration et du nombre de bains. 2/ Étude de l'agressivité de lavage : l'utilisation des ultrasons par l'effet de la variation de la pression locale, induit un phénomène mécanique de cavitation des membranes pilaires ce qui conduit par la suite à une mobilisation de la fraction interne en traces éléments de la matrice pilaire (3).

Conclusion : le lavage des cheveux avec de l'acide nitrique $10 \%$ à quatre reprises semble être la procédure de lavage la plus adaptée pour l'élimination de la majorité des éléments traces d'origine externe qui se sont déposés sur la tige pilaire, sans être agressive au point de toucher à l'intégrité de la matrice.

\section{Références :}

1. Pineau A. Contribution à l'étude de l'élimination des éléments traces par les produits de la sécrétion pileuse. Thèse d'Etat, Nantes 1987: 125.

2. Lekouch N. et coll. The science of the total environment 1999; 243/244 : 323-8.

3. Akalin M. et coll. Effects of ultrasonic energy on the wash fatness of relative dyes. Ultrasonics $2004 ; 42$ : 161-4. 


\section{Potentialités biologiques des épices utili- sées dans la cuisine marocaine : activité antibactérienne, toxicité, et impacts bio- chimique et histologique}

\section{J. EL MALTI $^{(1)}$, D. MOUNTASSIF ${ }^{(2)}$, H. AMAROUCH ${ }^{(1)}$}

(1) Laboratoire de Microbiologie, Pharmacologie, Biotechnologie et Environnement ;

(2) Laboratoire de Biochimie et Biologie Moléculaire, Université Hassan II, Casablanca, Maroc.

Objectif : étude de l'activité antibactérienne de 11 extraits éthanoliques des épices utilisées dans la cuisine marocaine testés sur 17 souches bactériennes gram positif et négatif susceptibles de contaminer les aliments. La toxicité des épices présentant un grand pouvoir antibactérien, a été testée sur des souris Swiss albino.

Méthodes : l'activité antibactérienne a été déterminée par la méthode de dilution sur milieu gélosé (1). Des souris Swiss albino ont été gavées par diverses doses d'extraits d'épices (Elletaria cardamomum, Myristica fragrans, Laurus nobilis), ensuite les dosages des biomarqueurs de stress (catalase et malondialdéhyde), des marqueurs subcellulaires (Succinate déshydrogénase et Glyceraldéhyde 3-phosphate déshydrogénase) et des paramètres cliniques sanguins (CPK, TGO, TGP, Urée et créatinine) ont été réalisés. Cette étude a été complétée par l'analyse de l'impact de ces extraits sur la numération des globules blancs et par l'analyse histopathologique (2) au niveau du cœur, foie, cerveau et intestin des souris témoins et traitées.

Résultats : l'activité antibactérienne présente des variations significatives suivant l'épice et la dose utilisées avec des CMI $<2.34 \mathrm{mg} / \mathrm{mL}$. L'étude de la toxicité a montré qu'à des doses élevées de ces extraits, des perturbations métaboliques sont observées accompagnées d'un stress oxydatif et des dysfonctionnements hépatiques et cardiaques.

Conclusion : cette étude confirme les propriétés antibactériennes des extraits éthanoliques d'épices utilisées dans la cuisine marocaine, ce qui suggère leur emploi éventuellement comme bioconservateurs dans l'industrie agro-alimentaire. Cependant, la consommation de ces épices doit être modérée pour éviter tout effet toxique d'une surdose.

\section{Références :}

1. Gulluce, M. et coll. In vitro antibacterial, antifungal, and antioxidant activities of the essential oil and methanol extracts of herbal parts and callus cultures of Satureja hortensis L. J. Agric. Food Chem. 2003; 51 : 3958-65.

2. Cau P. et coll. Cours de biologie cellulaire. Ellipses, Paris, 1996 ; 73 pp.
Impact du ciprofibrate (Lipanor®) sur la gerboise : étude biochimique, clinique et histologique

D. MOUNTASSIF ${ }^{(1)}$, M. KABINE(1), K. MOUNCHID ${ }^{(2)}$, K. MOUNAJI ${ }^{(3)}$, M.C. MALKI ${ }^{(4)}$, N. LATRUFFE(4), M.S. EL KEBBAJ ${ }^{(1)}$

(1) Laboratoire de Biochimie et Biologie Moléculaire ;

(2) Laboratoire d'Histologie et Embryologie ;

(3) Laboratoire de Physiologie et Génétique Moléculaire, Université Hassan II, Faculté des Sciences, Casablanca, Maroc ;

(4) Laboratoire de Biologie Moléculaire et Cellulaire (GDR-CNRS $\mathrm{n}^{\circ} 2583$ ), Université de Bourgogne, Faculté des Sciences, Dijon, France.

Objectif : le ciprofibrate est l'un des médicaments hypolipémiants utilisés par les patients dyslipidémiques afin de diminuer le risque d'athérosclérose. Notre étude a pour objectif l'analyse de l'impact de ce médicament chez la gerboise (animal doué d'une grande capacité à ne pas développer d'hépatomégalie une fois traité par certains médicaments contrairement au rat, à la souris et à l'homme).

Méthodes : l'effet de ce médicament a été étudié chez la gerboise (Jaculus orientalis) par une injection quotidienne de $3 \mathrm{mg} / \mathrm{kg}$ par voie intrapéritonéale pendant 4 semaines. Le dosage des enzymes cliniques, des biomarqueurs de stress, des marqueurs subcellulaires ainsi que l'analyse histologique ont été effectués.

Résultats : les résultats obtenus ont montré que le ciprofibrate a induit au niveau du foie un stress oxydatif très significatif et l'étude microscopique a montré une nécrose hépatique et une hypercondensation chromatinienne au niveau du cerveau.

Conclusions : cette étude ainsi que d'autres travaux ont confirmé que l'ingestion du ciprofibrate pendant de longues durées pourrait provoquer des dysfonctionnements hépatiques et nerveux. Ce qui nous oblige à être bien vigilants à la fois dans la prescription et l'utilisation de ce médicament.

Dosage de la paraphénylène diamine (PPD) dans le sang par chromatographie gazeuse couplée à la spectrométrie de masse (CPG-SM)

Y. BOUSLIMAN ${ }^{(1)}$, T. BASSET ${ }^{(1)}$, J.P. GAY-MONTCHAMP(1) $^{(1)}$ A.A. ZEGGWAGH ${ }^{(2)}$, Y. CHERRAH ${ }^{(3)}$, M. OLLAGNIER ${ }^{(1)}$

(1) Laboratoire de Toxicologie, Saint Etienne, France ;

(2) Service de Réanimation Médicale et de Toxicologie Clinique, CHU Ibn Sina, Rabat, Maroc ;

(3) Laboratoire de Pharmacologie, Rabat, Maroc.

Objectif : la paraphénylène diamine (PPD) est une amine aromatique utilisée comme teinture capillaire 
responsable d'intoxication mettant en jeu le pronostic vital. $(1,2)$. L'objectif de ce travail est la mise au point et la validation d'une technique de dosage de la PPD dans le sang.

Méthode : la méthode est basée sur le dosage de la PPD (pureté $>97.0 \%$ ) dans le sang par chromatographie gazeuse couplée à la spectrométrie de masse après extraction liquide-liquide. Le protocole de validation a englobé l'étude du coefficient d'extraction, le domaine de mesure, l'exactitude, la répétabilité et la reproductivité. (3)

Résultats : la courbe d'étalonnage est linéaire entre 98 est $1350 \mu \mathrm{g} / \mathrm{L}$ (coefficient de corrélation $\mathrm{r}=0.999$ ), la limite de détection est de l'ordre de $37 \mu \mathrm{g} / \mathrm{l}$, l'exactitude : 87 à $107 \%$, la répétabilité : $\mathrm{CV}=2.3$ à $9.7 \%$ et la reproductibilité : $\mathrm{CV}=4.4$ à $9.8 \%$ sont acceptables.

Conclusion : une méthode de dosage de la PPD dans le sang par chromatographie en phase gazeuse couplée à la spectrométrie de masse est mise au point. La validation de la méthode a démontré une bonne linéarité, une bonne exactitude, une limite de quantification basse, une répétabilité et une reproductibilité conformes aux normes.

\section{Références :}

1. Zeggwagh A. et coll. Myocardite toxique due à la paraphénylène diamine a propos de deux cas. Réan Urg. $1996 ; 5(6): 699-703$.

2. Zeggwagh A. et coll. Thrombus ventriculaire gauche et myocardite toxique induite par la paraphénylène diamine. Ann Fr Anesth Réanim. 2003 ; 22 : 639-41.

3. Nicolas O. et coll. Stratégie de validation de méthodes de dosage en bioanalyse en vue d'études pharmaceutiques et toxicologiques. Ann. Toxicol. Anal. 2004 ; XVI (2) : 118-27.

\section{Prévalence de la drogue, du tabac et de l'alcool chez les jeunes en milieu scolaire}

A. STAMBOULI, L. MERGAOUI-RHOLI, N. EL KARNI, M. WINDY, F. ZALAGH, R. AGHANDOUS

Société Marocaine de Toxicologie Clinique et Analytique (SMTCA), Rabat, Maroc

Objectif : l'enquête entreprise en juin 2006 par la SMTCA porte sur l'usage de la drogue, du tabac et de l'alcool chez les jeunes en milieu scolaire. Elle tente de rapporter des données exhaustives sur la toxicomanie, sur les produits psychotropes consommés par les jeunes, l'âge moyen de la première prise, les sources d'approvisionnement et conditions de consommation (seul, en groupe, fêtes,..), la taille de la population jeune concernée par ce phénomène et ce afin de proposer en retour des campagnes de sensibilisation et de prévention adaptées.

Méthode : d'après le rapport 2005 de l'ONUDC, l'âge d'initiation au tabac, à l'abus et à l'injection de drogues, a enregistré une baisse dont les causes sont à rechercher dans le manque de la cohésion sociale et aussi à cause de la grande disponibilité des différentes variétés de drogues à des prix qui restent abordables pour les jeunes. Cette toxicomanie se traduit dans le milieu scolaire par l'absentéisme, le décrochage scolaire, la violence physique et verbale, la dégradation des locaux d'établissements publics, l'agression des enseignants etc.

Pour mener son enquête, la SMTCA a réalisé un échantillonnage aléatoire parmi les jeunes scolarisés dans les écoles, lycées et collèges des grandes agglomérations de Casablanca, Rabat, Salé, Mohammédia, et Témara. Le questionnaire élaboré à cet effet, est anonyme, non invasif et porte sur dix questions rédigées en termes simples arabes et français de sorte à ce que la durée de réponse n'excède généralement pas $10 \mathrm{~min}$.

Résultats : à ce jour, le nombre des jeunes interrogés est de 6231 , réparti en $54 \%$ de filles et $46 \%$ de garçons, appartenant à 30 écoles, lycées et collèges différents. Le taux de participation exprimé en nombre de réponses exploitables/nombre de consultations, est de $89,5 \%$. Le dépouillement des réponses révèle que $17 \%$ des garçons interrogés auraient consommé au moins une fois de la drogue contre $4,2 \%$ des filles. L'âge au cours duquel ces jeunes sont plus tentés par l'usage de la drogue se situerait entre 16 - 18 ans pour les garçons (71\%) et entre 17 - 19 ans pour les filles. Le cannabis est de loin la drogue la plus couramment consommée $(50 \%)$ par les jeunes qui s'adonnent aux psychotropes, $12 \%$ font usage de cocaïne et $3 \%$ d'héroïne. D'autres substances achetées sur le marché sont également employées, amphétamines, solvants, divers médicaments, etc. La majorité de ces jeunes $(80 \%)$, se livre à la consommation entre amis contre une minorité $(13 \%)$ qui préfère s'isoler. Il est facile de se procurer de la drogue au marché noir pour $66 \%$ des personnes interrogées. L'accoutumance à la cigarette est développée chez $4,9 \%$ de garçons et $1,1 \%$ de filles. Cette tendance est respectée également chez les fumeurs occasionnels qui sont composés respectivement de 8,3\% de garçons et $2,8 \%$ de filles. La consommation régulière du tabac est enregistrée fortement pour la tranche d'âge 14 - 16 ans pour les filles et 17 - 18 pour les garçons.

L'initiation à la drogue et au tabac se ferait pour les deux sexes dès l'âge de 12 ans. Les réponses relatives à la consommation de l'alcool ne sont pas exploitables.

Conclusion : les résultats préliminaires de cette enquête démontrent clairement la tendance de la consommation de la drogue et du tabac chez les jeunes scolarisés dans les établissements publics et privés et qui reste légèrement inférieure à celle enregistrée dans les pays industrialisés. Plus de $10 \%$ des jeunes s'adonnent à la drogue. Les usagers du tabac chez cette population jeune représentent $17 \%$ (13,2\% garçons et 3,9\% filles). Compte tenu de l'effet addictif rapide de la cigarette, une intervention antitabac intensive et précoce auprès des fumeurs débutants ou occasionnels pourrait signifier des gains sur le plan de la lutte contre la toxicomanie. 


\section{Communications affichées}

\section{Intoxication aigues admises en réanima- tion : à propos de 1000 cas}

\author{
M. AIT EL CADI ${ }^{(1)}$, Y. KHABBAL ${ }^{(1)}$, Y. CHERRAH ${ }^{(1)}$, \\ N. MADANI ${ }^{(2)}, \mathrm{K} . \mathrm{ABIDI}^{(2)}, \mathrm{R} . \mathrm{ABOUQAL}(2)$, \\ A. A. ZEGGWAGH ${ }^{(2)}$
}

(1) Département de Pharmacologie-Toxicologie Faculté de Médecine et de Pharmacie Rabat ;

(2) Service de Réanimation Médicale et de Toxicologie Clinique, CHU Ibn Sina, Rabat, Maroc.

Introduction : les intoxications aigue (IA) sont une cause fréquente de consultation aux urgences. Elles sont dues à une exposition aigue, volontaire ou accidentelle, à un ou plusieurs toxiques. Le but du travail est de tracer le profit épidémiologique et pronostique de ces intoxications admises dans le service de réanimation médicale.

Méthodes : il s'agit d'une étude rétrospective étalée sur 14 ans (1992-2006) et incluant toutes les IA admises au service de réanimation médicale. Les intoxications par caustiques et survenant chez les patients de moins de 14 ans ont été exclues. Ces intoxications étaient diagnostiquées sur les données de l'anamnèse, de l'examen clinique et de l'analyse toxicologique. Les données démographiques, cliniques, para cliniques, évolutifs et thérapeutiques ont été recueillies à l'admission puis analysées. Les résultats sont exprimés en chiffre, en pourcentage ou en moyenne \pm écart type. Le groupe des patients décédés et celui des survivants sont comparés. Pour l'analyse univariée, les variables quantitatives sont comparées par le test de Student, et les variables qualitatives par le Chi2 ou le test exact de Fisher. L'analyse multivariée a fait appel au modèle de régression logistique pas a pas ascendant.

Résultats : mille patients ont été inclus correspondant à une incidence des IA de $21 \%$ dans notre unité. L'age moyen était de $28,4 \pm 8,5$ ans. $69 \%$ des intoxiqués étaient de sexe féminin. Le délai entre l'intoxication et l'admission était de 10 heures. L'intoxication était volontaire dans $92 \%$ des cas. L'indice de gravite simplifié (IGS II) était égale a $17 \pm 11$. Les produits agricoles étaient incriminés dans $40 \%$, les médicaments dans $35 \%$ et les produits industriels dans $23 \%$ des cas. Le profit clinique des patients comprenait des troubles de la conscience dans $50 \%$, des convulsions dans $15 \%$, un état de choc dans $10 \%$ et les signes digestifs dans $20 \%$ des cas. $45 \%$ des intoxiques avaient nécessite une incubation endotrachéale avec une durée moyenne de ventilation de $4+3$ jours. La mortalité globale était de $7 \%$. La mortalité des intoxications médicamenteuses était significativement plus basse que celle des intoxications non médicamenteuses ( $2 \%$ vs $11 \% ; p<0,05$ ). Les facteurs pronostiques indépendants en terme de mortalité étaient les défaillances circulatoire et respiratoire.
Conclusion : les IA admises en réanimation concernent essentiellement l'adulte jeune de sexe féminin et sont en majorité volontaires. Elles sont dominées par les produits agricoles et les médicaments. La mortalité est élevée, principalement liée a certains toxiques dangereux en vente libre (paraphénylène diamine et phosphure d'aluminium), incitant à leur interdiction ou à leur réglementation stricte. Le pronostic semble lié à l'atteinte cardiovasculaire et respiratoire.

\section{Intoxication par l'insuline}

\section{Y. KHABBAL $^{(1)}$, M. AIT EL CADI ${ }^{(1)}$, Y. CHERRAH ${ }^{(1)}$, F. AJDI $^{(2)}$, I. BENABBAD ${ }^{(2)}$, A.CHRAIBI ${ }^{(2)}$}

(1) Département de Pharmacologie-Toxicologie Faculté de Médecine et de Pharmacie, Rabat, Maroc ;

(2) Service d'Endocrinologie, Diabétologie et Nutrition, CHU Ibn Sina, Rabat, Maroc.

Introduction : les intoxications par l'insuline niées par le patient appelées communément hypoglycémies factices sont caractérisées par des symptômes physiques et psychiques provoqués volontairement par le sujet luimême. Nous rapportons dans ce travail le cas de deux patientes présentant une intoxication volontaire par l'insuline non dans un but d'autolyse mais dans un contexte d'hypoglycémie factice.

Observations : il s'agit de deux patientes qui avaient présenté des hyperglycémies à jeun et post prandiales. Le diagnostic de diabète avait été retenu et l'insulinothérapie fut instaurée. L'évolution a été marquée par des malaises hypoglycémiques qui sont survenus malgré l'arrêt de l'insulinothérapie. L'HGPO et des taux HbA1c étaient normaux alors que les glycémies capillaires étaient perturbées. C'est grâce à la découverte de points d'injections au niveau des bras avec une insulinémie élevée et une glycémie concomitante discordante que les diagnostics de diabète factice et d'intoxication par l'insuline ont été posés. Une expertise psychiatrique a alors été faite ce qui a confirmé notre hypothèse diagnostique.

Conclusion : les hypoglycémies factices sont des intoxications dont le diagnostic est difficile à établir. Un dosage de l'insulinémie et des hypoglycémiants oraux s'impose devant toute hypoglycémie chez les patients ayant un profil psychologique particulier.

\section{Intoxication par les corticoïdes}

M. AIT EL CADI ${ }^{(1)}$, Y. KHABBAL ${ }^{(1)}$, Y. CHERRAH ${ }^{(1)}$, I. BENABBAD $^{(2)}$, F. AJDI ${ }^{(2)}$, A. CHRAIBI ${ }^{(2)}$

(1) Département de Pharmacologie- Toxicologie. Faculté de Médecine et de Pharmacie, Rabat, Maroc ;

(2) Service d'Endocrinologie, Diabétologie et Nutrition, CHU Ibn Sina, Rabat, Maroc.

Objectif : la notion de prise de corticoïdes doit être vérifiée devant toute exploration d'un syndrome de Cushing compte tenu du danger que représente leur 
mauvaise utilisation. Le but de notre travail est d'étudier les cas de syndrome de Cushing secondaires à l'intoxication chronique par les corticoïdes.

Méthode : il s'agit d'une étude rétrospective incluant tous les syndromes de Cushing admis au service d'Endocrinologie. L'étude a porté sur l'anamnèse, l'examen clinique, les tests biologiques, les dosages hormonaux et sur la radiologie. Les résultats ont été exprimés en pourcentage ou en moyenne \pm écart type.

Résultats : huit patients ont été inclus, l'âge moyen est de $46 \pm 14$ ans. $75 \%$ des intoxiqués étaient de sexe féminin, tous présentaient les signes cliniques d'un syndrome de Cushing évident (obésité facio-tronculaire, vergetures pourpres, ecchymoses, asthénie...). La sécrétion cortisolique était effondrée dans tous les cas avec une moyenne de $18.6 \pm 12 \mu \mathrm{g} / \mathrm{l}$. Le test au synachtène était positif dans $80 \%$ des cas avec une élévation moyenne du cortisol de $140 \pm 80 \mu \mathrm{g} / \mathrm{l} .62 \%$ des patients présentaient des signes d'ostéoporose. La prise de corticoïdes a été évoquée devant le terrain particulier des patients à savoir que $100 \%$ avaient des antécédents d'asthme de durée variable. La voie orale était utilisée pour la prise des corticoïdes dans $100 \%$ des cas. De plus cette prise était anarchique. Les corticoïdes impliqués sont la prédnisone, la prednisolone, la méthyl prednisolone et la bêta-méthasone.

Conclusion : le syndrome de Cushing iatrogène réalise un tableau clinique de syndrome de Cushing associé à un tableau biologique d'insuffisance surrénalienne. C'est le résultat d'une intoxication chronique par les corticoïdes utilisés à long terme et de manière anarchique, nécessitant une prise de conscience de la part des patients et du personnel de santé.

\section{Intoxication par le sulfure d'hydrogène}

\section{N. MEFTAH, A. EL KHOLTI}

Service de santé au travail, CHU Ibn Rochd, Casablanca, Maroc

Objectif : l'hydrogène sulfuré $\left(\mathrm{H}_{2} \mathrm{~S}\right)$ est un gaz toxique, inflammable et explosif, rencontré en milieu industriel. Sa toxicité dépend du temps d'exposition et de la concentration atmosphérique. L'objectif de notre travail est d'étudier la toxicité de ce gaz et de préciser la clinique, le traitement et la prévention.

Résultats : l'hydrogène sulfuré est un puissant irritant de l'œil et des voies respiratoires (1), à l'origine d'un œdème aigue pulmonaire lésionnel. Les formes suraiguës sont caractérisées par le décès par arrêt cardio-respiratoire quasi immédiat. Le traitement repose essentiellement sur des mesures de réanimation symptomatiques. La prévention comporte des mesures techniques collectives, des moyens de protection individuelle, des règles d'hygiène générale, l'évaluation et la surveillance de l'exposition.

Conclusion : l'hydrogène sulfuré est un gaz mortel à concentration atmosphérique élevée. Sa toxicité est multiple. Des mesures de sécurité sont à préconiser aussi bien dans le milieu industriel qu'au cours du nettoyage de zones confinées de décomposition organique.

\section{Référence :}

1. Lauwerys R.R. Gaz et vapeurs irritants et asphyxiants. In : Toxicologie industrielle et intoxications professionnelles, Masson, Paris, 2003 ; 530-88.

\section{Conduite à tenir devant une intoxication professionnelle aigue au monoxyde de carbone}

B. RAJI, I. MIMOUNI, Y. EL AOUDI, A. EL KHOLTI

Service de santé au travail CHU Ibn Rochd, Casablanca, Maroc

Objectif : l'objet de ce travail est de décrire les différentes manifestations cliniques liées à l'intoxication au $\mathrm{CO}$, la conduite à tenir sur les lieux de l'accident et après transport à l'hôpital et les différents moyens de prévention et de réparation.

Méthodes : étude bibliographique sur les intoxications professionnelles au $\mathrm{CO}$.

Résultats : l'oxyde de carbone est un gaz incolore, inodore utilisé en métallurgie de différents métaux et dans l'industrie chimique pour la synthèse de nombreux composés comme le méthanol, l'acide acétique. L'intoxication aigue au CO peut entraîner une atteinte au système nerveux central et le syndrome neurologique retardé (SNR) ou syndrome post intervallaire qui est caractérisé par des manifestations neurologiques, comportementales et psychiatriques. On peut rencontrer des désordres moteurs (de type parkinsonien), des perturbations des fonctions du système nerveux autonome (incontinence urinaire et fécale) ou des fonctions cognitives, des pertes de la mémoire à court terme.

Conclusion : le CO est reconnu pour être responsable de nombreux accidents avec hospitalisation, séquelles, voire décès. L'organisation du travail doit chercher à limiter la l'exposition des salariés au CO lorsque celleci ne peut être complètement évitée et à déployer les moyens adaptés pour la protection individuelle et collective.

\section{Intoxication au maâjoune. Expérience du Centre Anti Poison du Maroc durant 14 ans}

G.H. JALAL, M. WINDY, F. CHAFIQ, N. RHALEM, M. BADRI, I. EMLALI, R. SOULAYMANI

Centre Anti-Poison et de Pharmacovigilance du Maroc

Objectifs : le maâjoune est un mélange pâteux dont la composition peut différer selon les régions mais la présence du chanvre indien est constante. D'autres composants peuvent être ajoutés comme les graines de pavot, jusquiame belladone et certains condiments : cardamome, maniguette, noix de muscade, cubèbe, 
cantharide (1, 2). La consommation du maâjoune semble être assez fréquente au Maroc. L'objectif de notre étude est de déterminer le profil épidémiologique des intoxications au maâjoune.

Méthodes : il s'agit d'une étude rétrospective concernant les cas d'intoxication au maâjoune déclarés à l'unité de toxicovigilance du Centre Anti Poison du Maroc (CAPM) sur 14 ans (1992 à 2005).

Résultats : au total 749 cas ont été déclaré à l'unité de toxicovigilance du Centre Anti Poison du Maroc (1.5\% de tous les cas d'intoxication). L'usage du maâjoune est très souvent volontaire dans un but toxicomanogène avec un pourcentage de 54,2\% mais peut être accidentel touchant en général les enfants dans 39\%. Des intoxications criminelles provoquées à des fins perverses peuvent aussi se rencontrer dans $3.2 \%$ des cas. Le sexe masculin est prédominant (sexe ratio $=7.6$ ). La symptomatologie est faite le plus souvent de signes neuropsychiatriques. Le traitement est en général symptomatique et l'évolution reste favorable dans $92 \%$.

Conclusion : l'ignorance de la gravité de la toxicité de ses composants peut avoir des répercutions désastreuses sur la santé en cas de surdosage et multi-usage mais aussi un impact socio-économique important.

\section{Références :}

1. Duke James A. Handbook of medicinal herbs. CRC press, 2000 ; 96-7.

2. Bellakhder J. La pharmacopée marocaine traditionnelle, Médecine arabe ancienne et savoirs populaires. Ibis Press, 1997 ; 132-3.

3. Vigneau C. Plantes médicinales, Masson. 1989 ; 80101 .

\section{Épidémiologie des intoxications au Maroc. Analyse des fiches de toxicovigi- lance (1983-2001)}

N. ATTAZAGHARTI ${ }^{(1)}$, I. SEMLALI ${ }^{(2)}$, A. SOULAYMANI ${ }^{(1)}$, A. MOKHTARI ${ }^{(1)}$, R. SOULAYMANI BENCHIKH ${ }^{(2)}$

(1) Laboratoire de Génétique et Biométrie, Université Ibn Tofail, Kénitra, Maroc ;

(2) Centre Antipoison et de pharmacovigilance du Maroc, Rabat, Maroc.

Introduction : les accidents liés aux intoxications sont un véritable fléau et un grand problème de santé publique. La fréquence et les conséquences qui en résultent, montrent la gravité et l'ampleur de ce problème au monde. Au Maroc, les intoxications qui sont fréquentes ont des conséquences économiques et sociales très importantes. En effet, chaque année des patients décèdent à la suite de cette pathologie.

Objectif : l'objectif de notre travail est d'analyser et interpréter les fiches de toxicovigilance du centre antipoison du Maroc, sur une période de 4 années, de tracer le profil épidémiologique de cette pathologie, de définir les facteurs de risque dans le but de contribuer à réduire l'incidence des intoxications et d'améliorer le comportement de la population et des professionnels vis-à-vis des toxiques.

Matériel et méthodes : nous rapportons une étude épidémiologique rétrospective sur 4 ans (1998-2001) à partir des données des fiches de déclaration de toxico vigilance. L'analyse a consisté à faire une étude descriptive et analytique globale des cas.

Résultats : 7754 cas d'intoxication ont été colligés entre 1998 et 2001, les intoxications par piqûres de scorpion occupent la première place par rapport à l'ensemble des intoxications. Elles représentent 37,7\%, suivies par les médicaments avec $30,2 \%$, le $\mathrm{CO}$ avec $28,4 \%$ et enfin les intoxications par les plantes $3,7 \%$. Différents paramètres ont été étudiés. Ainsi, la classe d'âge la plus touchée est comprise entre 20 et 30 ans avec un pourcentage de 25,4. Parmi la population intoxiquée, ce sont les femmes qui paraissent les plus touchées avec $62,8 \%$, alors que les hommes ne sont affectés qu'avec un pourcentage de 37,2. Par conséquent, le sexe ratio est de 0,59 . Quant à l'origine de la population intoxiquée, elle est de $60 \%$ urbaine et de $40 \%$ rurale. S'agissant du lieu d'intoxication, la majorité des intoxications surviennent à domicile avec un pourcentage de $88,6 \%$. De manière générale, l'exposition aux toxiques est unique dans $93,4 \%$ des cas. Ainsi, le pourcentage des patients arrivant au niveau des différentes structures sanitaires durant ces années, a montré que les hôpitaux reçoivent plus de patients intoxiqués $(89.7 \%)$ par rapport aux autres structures sanitaires.

Conclusion : l'exploitation des données a permis de montrer la gravité et l'ampleur du problème des intoxications au Maroc, par sa fréquence et son impact sur les victimes.

Les conséquences économiques et sociales sont loin d'être négligeables et méritent plus d'attention des responsables de la santé publique dans notre pays.

\section{Étude de l'impact des toxines paraly- santes sur le métabolisme cellulaire de la souris : Effets biochimiques et histolo- giques}

\section{N. TAKATI $^{(1)}$, D. MOUNTASSIF ${ }^{(2)}$, M. BLAGHEN ${ }^{(1)}$}

(1) Laboratoire de Microbiologie, Pharmacologie, Biotechnologie et Environnement ;

(2) Laboratoire de Biochimie et Biologie Moléculaire, Université Hassan II, Faculté des Sciences, Casablanca, Maroc.

Objectif : étude des effets biochimiques et histologiques des toxines paralysantes sur le métabolisme cellulaire de la souris.

Méthodes : les extraits toxiques sont préparés à partir de la chair de la coque d'Acanthocardia tuberculatum 
selon la méthode officielle de l'A.O.A.C (1). Ils ont été injectés par voie intrapéritonéale à des souris Swiss albino quotidiennement pendant 7 jours à une dose équivalente à $10 \mu \mathrm{g}$ de STX/100g de chair. Les dosage des biomarqueurs de stress (catalase et malondialdéhyde), des marqueurs subcellulaires (Succinate déshydrogénase et Glyceraldehyde 3-phosphate déshydrogénase) et des paramètres cliniques sanguins (CPK, TGO, TGP, Urée et créatinine) ont été réalisés. En parallèle, une étude histopathologique de l'effet des toxines paralysantes sur les différents organes de la souris a été effectuée (2).

Résultats : le dosage des biomarqueurs tissulaires montre des perturbations métaboliques avec des dysfonctionnements surtout hépatiques et cardiaques. L'analyse au microscope optique des coupes histologiques des divers organes prélevés chez la souris confirme les résultats des dosages des ces marqueurs.

Conclusions : nos résultats ont permis de conclure que les perturbations observées au niveau cardiaque s'expliquent surtout par le fait que les toxines paralysantes sont des neurotoxines qui provoquent la mort par insuffisance cardiaque.

\section{Références :}

1. AOAC. Paralytic shellfish poison, biological method, final action1990. In: AOAC (ed.), Official Methods of Analysis, 15th ed., Arlington, VA, method $\mathrm{n}^{\circ}$ 959.08.

2. Cau P., Seïte R. Cours de biologie cellulaire. Ellipses, Paris, 1996.

\section{Situation épidémiologique des morsures de serpent au Maroc (2001-2005)}

F. CHAFIQ, M. WINDY, G. JALAL, M. BADRI, I. SEMLALI, N. RHALEM, R. SOULAYMANI

Centre Anti Poison et de Pharmacovigilance du Maroc

Objectif : au Maroc les envenimations par morsure de serpent (MS) constituent un sujet d'inquiétude pour les populations et une préoccupation pour le personnel de santé. Le but de notre étude épidémiologique est d'évaluer l'incidence et la gravité des morsures de serpent (MS), les facteurs de risques, les aspects cliniques et évolutifs.

Méthodes : étude rétrospective sur 5 années (20012005) de tous les cas d'envenimation par morsure de serpent déclarés par les différentes provinces médicales au département de la toxicovigilance du Centre Anti Poison du Maroc (CAPM). L'analyse des données est réalisée sur le logiciel Epi-info.

Résultat : durant la période s'étalant de 2001 à 2005, 428 cas de MS ont été déclarés au CAPM soit une incidence cumulée de 0,02 pour 100000 habitants par an. Les MS représentent $53 \%$ de l'ensemble des morsures par animaux venimeux (scorpion exclu). Le nombre de morsures augmente au cours de la période allant de mars à septembre avec un pic de juin à septembre. La région de Souss Massa Draa occupe la première place soit 40,4\% des cas d'envenimation suivi par la région Marrakech-Tensift $(28,5 \%)$ et au troisième rang la région de Meknes-Tafilalt (16,3\%). L'âge moyen des victimes est de 28,5 ans \pm 19 ans, le sex-ratio (male/femelle) est de 1,7. Les enfants (15 ans représentent $34 \%$. Dans $86 \%$ des cas les victimes sont d'origine rurale. Le taux d'envenimation est de $94,2 \%$. La traduction clinique est polymorphe avec prédominance par ordre de fréquence décroissant : symptomatologie digestive, neurologique cardiovasculaire respiratoire et cutanée. La létalité générale est de 5,6 \% (24 décès). La létalité spécifique aux enfants (15 ans est importante : elle est de 9,5\%. L'identification de l'espèce de serpent agresseur n'a été faite dans aucun cas. Le sérum antivenin quoique son efficacité a été prouvée $(1,2)$ n'a été indiqué dans aucun cas du fait de sa non disponibilité.

Conclusion : l'envenimation par morsure de serpent constitue un problème de santé publique, la létalité est importante. Les 3 régions les plus touchées sont Souss Massa daraa, région de Marrakech-Tensift, et la région de Meknes-Tafilalt. Pour diminuer la morbidité et la mortalité par envenimation de serpent, la disponibilité de sérum adapté aux espèces les plus venimeuses au Maroc devient une priorité.

\section{Références :}

1. Audebert F. et coll. Envenoming by viper bites in France : clinical gradation and biological quantification by ELISA. Toxicon, $1992 ; 30: 599-609$.

2. Chippaux J.P. L'envenimation ophidienne en Afrique : épidémiologie clinique et traitement. Ann. IP / actualités $1999 ; 10: 161-71$.

\section{Profil épidémiologique des intoxications au Maroc : données de toxicovigilance 1992-2005}

I. SEMLALI, S. BENLARABI, G. EL OUFIR, M. BADRI, R. SOULAYMANI-BENCHEIKH

Centre Anti Poison et de Pharmacovigilance du Maroc, Rabat, Maroc

Introduction : le Département de Toxicovigilance (TV) travaille selon trois actions qui sont la collecte, la validation et la prise de décision. La toxicovigilance dispose d'un système d'information jouant le rôle d'un observatoire en suivant le profil épidémiologique des différents types d'intoxications dans les différentes provinces du royaume.

Objectifs : décrire le profil épidémiologique des intoxications au Maroc et de suivre l'évolution des différents indicateurs par tranche de 5 ans.

Matériels et méthodes : nous rapportons une étude épidémiologique descriptive sur 14 ans (1992-2005) et selon 3 périodes $(\mathrm{p})$ intercalées par 2 actions marquées: une $1^{\text {ère }}$ période (p1: 1992 à 1995) qui est suivie par une 
relance active des déclarations, une $2^{\text {ème }}$ période $(\mathrm{p} 2$ : 1996 à 2000) après laquelle il y a eu instauration d'un système d'information spécifique aux piqûres et envenimations scorpioniques (PES), et une $3^{\text {ème }}$ période (p3: 2001 à 2005) où les PES ne font plus partie du système standard des déclarations. Cependant, tous les cas de piqûres et envenimations scorpioniques (soit 12608 cas) ont été soustraits de la banque de données de toxicovigilance (BDTV) pour ne pas biaiser l'analyse. La collecte des cas a été opérée à partir des fiches de déclaration des cas d'intoxication (FTV) mises à la disposition des structures sanitaires et transmises régulièrement au Centre Anti Poison du Maroc pour les saisir dans la banque de données de toxicovigilance (BDTV) (données non encore publiées).

Résultats et discussion : durant les 14 dernières années, on a colligé 41.220 cas d'intoxications (excepté les PES). Les intoxications par les animaux (excepté le scorpion) représentent entre $4,4 \%$ et $6 \%$ parmi les autres intoxications (toute cause confondue). La majorité des toxiques ont gardé leur classement habituel, sauf pour les aliments qui sont devenus au $1^{\text {er }}$ plan dans les deux périodes P2 (26\%) et P3 (37\%), aussi les produits gazeux ont pris la $2^{\text {eme }}$ place $(20,4 \%)$ contre les médicaments qui ont reculé pour être classés en $3^{\text {ème }}$ position $(14,2 \%)$ dans la période 3 . Le Grand Casa garde toujours la 1 ère place dans les déclarations des cas dans les 3 périodes. Les régions: Marrakech-Tensift-Al Houz, Tadla-Azilal et l'Oriental ont pris le devant dans les déclarations. Les régions : Tanger-Tétouan, SousMassa-Draa, Chaouia-Ourdigha et Rabat-SaléZemmour-Zear déclarent encore moins qu'auparavant. Il y a prédominance du sexe féminin avec un âge moyen autour de 22-23 ans + un écart type autour de 14 ans pour les 3 périodes. Les intoxications à domicile sont toujours prédominantes, suivies par le milieu publique et plus rarement le lieu de travail dans les 3 périodes. Les circonstances ont gardé le même ordre de classement dans les 3 périodes, avec une prédominance des circonstances accidentelles, suivies par les suicidaires et très rarement les autres types. La voie orale est la plus prédominante, suivie par la voie d'inhalation et plus rarement percutanée dans les 3 périodes. L'exposition est unique dans la majorité des cas, rarement itérative dans les 3 périodes, exceptionnellement chronique dans la période $1(0,2 \%)$ voir presque inexistante pour les périodes $\mathrm{P} 2$ et $\mathrm{P} 3$. La symptomatologie gastro-intestinale est la plus prédominante, suivie par les signes neurologiques, respiratoires puis cardio-vasculaires. Les autres symptômes sont exceptionnels voir inexistants dans les périodes $\mathrm{P} 2$ et $\mathrm{P} 3$. Le lavage gastrique (LG) vient en 1 èr position, suivi par les vomissements provoqués (VP), très rarement l'épuration pulmonaire (EP) et exceptionnellement l'épuration rénale (ER) pour les 3 périodes. Le LG est plus fréquemment utilisé $(82,7 \%)$ que les VP $(13,1 \%)$ dans la période 3 . Dans les 3 périodes, l'évolution était dans la majorité des cas favorable, certains avaient présenté des com- plications $(<0,6 \%$ des cas $)$, mais on note toujours des cas de décès non négligeables (entre 1,4 et 2,2 \% des cas). Cependant, l'évolution reste inconnue (entre 17 et $25 \%$ ), du fait du transfert des patients vers un autre service ou une autre structure sanitaire et par l'absence de feed-back. La létalité par Takaout reste au $1^{\text {er }}$ plan (P1 : 71,4\%, P2 : 91,4\%), avec une nette diminution dans la période P3 (12,9\%). La létalité par les animaux, les produits industriels et les aliments a augmenté surtout dans la période P3. La part des pesticides dans les décès reste toujours au 1er plan dans les 3 périodes avec une augmentation croissante d'une période à l'autre (P1 : 25,1\% ; P2 : 33,5\% et P3 : 37,0\%). La part des plantes et celle des médicaments dans les décès a beaucoup régressé dans les périodes $\mathrm{P} 2$ et $\mathrm{P} 3$, tandis que celle des animaux a doublé dans les périodes $\mathrm{P} 2$ $(13,2 \%)$ et $\mathrm{P} 3(12,5 \%)$ et celle des aliments a augmenté dans les périodes $\mathrm{P} 2(6,1 \%)$ et P3 $(5,5 \%)$. Les produits gazeux ont régressé dans la période $2(4,4 \%)$, pour reprendre la même part dans les décès que la $1^{\text {ère }}$ période (autour de 10\%).

Conclusion : l'étude descriptive du profil des intoxications par tranche de 5 ans (en moyenne), est un premier pas qui a donné une vue globale sur les intoxications au Maroc. Elle montre que le nombre des déclarations ne cesse d'augmenter, ceci grâce à la sensibilisation des professionnels de santé. Le profil général des intoxications a changé pour les médicaments, les aliments et les produits gazeux. Certains paramètres ont augmenté en pourcentage, d'autres ont diminué, cependant il faut choisir des indicateurs de suivi spécifiques pour chaque toxique et confirmer chaque changement par les tests de significativité. La centralisation et la gestion des intoxications par le Centre Anti Poison et de Pharmacovigilance du Maroc (CAPM), permettent de suivre le profil épidémiologique général des intoxications, ce qui aide à une meilleure réflexion pour une prise de décision adéquate. Le programme d'éducation, de prévention et de lutte anti-toxique doit être renforcé par le Ministère de la Santé pour diminuer la morbidité et la mortalité causées par l'intoxication qui restent non négligeables.

\section{Ischémie cérébrale secondaire à une envenimation vipérine : à propos de deux cas et revue de la littérature}

\section{A. TIJANI, NEJMIH, MA. SAMKAOUI, MG. EL ADIB, S. YOUNOUS}

Service d'anesthésie-réanimation, CHU Med VI, Maroc

Les accidents d'envenimation constituent un problème de santé public dans certains pays, on recense environ 2700 espèces de serpents dont 300 sont venimeuses et potentiellement dangereuses pour l'homme. Elles sont responsables d'une grande mortalité et morbidité, avec 1000 morts rapportés chaque année en malaisie. Aux 
USA, 85000 morsures par an sont rapportées dont 18\% par serpent venimeux. En Afrique ces accidents touchent environ $0.5 \%$ de la population par an.

Nous rapportons deux observations cliniques de deux patientes admises dans un tableau d'ischémie cérébrale suite à une envenimation vipérine (grade III) dont l'évolution était fatale. Nos deux patientes ont présenté un accident vasculaire cérébral ischémique étendu compliqué d'oedème cérébral important engendré le décès dans un tableau d'engagement.

Les lésions ischémiques cérébrales causées par envenimation vipérine surviennent plus souvent que ce qui est rapporté dans la littérature. On pense que le mécanisme physiopathologique inclut une vasoconstriction et une ischémie au niveau cérébral causée par le venin ou par la formation de microthrombi suite à l'hypotension et à la coagulopathie induite par ce venin.

Le nombre d'envenimations vipérines rapportées chaque année au Maroc et le manque d'expérience du personnel médical et paramédical sont derrière les difficultés de prise en charge des malades victimes de morsures de vipères. L'éducation du grand public et des praticiens est essentielle pour fournir à tous, la connaissance nécessaire pour traiter ces malades.

\section{Évaluation de l'impact de la standardi- sation de la prise en charge sur le profil de l'intoxication à la PPD}

M. WINDY, G. JALAL, F. CHAFIQ, M. BADRI, N. RHALEM, I. SEMLALI, R. SOULAYMANI

Centre Anti-Poison et de Pharmacovigilance du Maroc

Objectifs : la paraphénylène diamine (takaout roumia) est une amine aromatique introduite au Maroc depuis 1970 (1). L'objectif de notre étude est de tracer un profil épidémiologique de l'intoxication à la paraphenylène diamine (PPD) et d'évaluer l'impact de la mise en place d'une conduite à tenir face à l'évolution de cette intoxication au cours des six dernières années.

Méthodes : il s'agit d'une étude rétrospective menée sur une période de six ans allant de 2000-2005. Les données sont recueillies sur les fiches de l'unité de toxicovigilance du Centre Anti-Poison et de pharmacovigilance du Maroc CAPM.

Résultats : durant la période allant de 2000-2005 le CAPM a recensé 289 cas d'intoxications à la paraphényléne diamine $(1.02 \%$ de l'ensemble des intoxications). Cependant, les intoxications à la PPD ont connu une diminution constante ces dernières années, elles sont passées de 62 cas pour l'année 2000 à 17 cas 2005. La femme est la plus touchée $81 \%$ par ce type d'intoxication avec un sexe ratio $(\mathrm{m} / \mathrm{f})$ de 0,24 . L'âge des intoxiqués se situe entre 1 et 70 ans avec une moyenne de 24.6 ans \pm 11.19 ; la tranche d'âge la plus touchée se situe entre 15 et 25 ans avec un pourcentage de $54.4 \%$. La tentative de suicide occupe la première place parmi les circonstances de survenue avec un pourcentage de $83.4 \%$, suivie de la circonstance accidentelle $9.4 \%$, criminelle $1.1 \%$ et toxicomaniaque $0.7 \%$. Dans $69 \%$ des cas, les patients étaient symptomatiques : avec des signes digestifs (55.7\%), respiratoires (21\%), neurologiques $(10.8 \%)$ et cardiovasculaires $(12.5 \%)$. L'évolution a été favorable dans $80.6 \%$, le décès est survenue dans $19.4 \%$.

Conclusion : grâce à la mise en place d'une conduite à tenir standardisée au niveau des milieux hospitaliers, des campagnes de sensibilisation et de la nouvelle réglementation de vente de ce produit, les intoxications à la PPD ont connu une nette diminution et leur prise en charge ont considérablement été améliorée.

\section{Références :}

1. Baud F. et coll. Rhabdomyolyse au cours d'une intoxication aigue par la paraphénylène diamine, $\mathrm{J}$. Tox. Med. $1984 ; 279-83$.

2. Belakhder J. Médecine arabe ancienne et savoir populaires. Pharmacopées traditionnelles. Ibis presse, 1997 ; 510-2.

3. Ashraf W. et coll. Systemic paraphenylen diamine poisoning: a case report and rewiew. Hum. Exp. Toxicol $1994 ;$ 167-70.

\section{Intoxications aigues aux organophos- phorés chez l'enfant}

\section{S. BICHRI, G. DRAISS, A. SAKHI, F. JENNANE,} B. SLAOUI, F. DEHBI

Service de pédiatrie 2 ; Hôpital d'Enfants, Casablanca, Maroc

Introduction : les intoxications aigues constituent de plus en plus un problème grave dans les pays en voie de développement du fait de leur fréquence et des difficultés de prise en charge. Les intoxications par les organophosphorés sont rares mais graves en raison de leur forte toxicité et de leur action systémique prédominante, représentant $3 \%$ des intoxications aigues chez l'enfant.

Objectifs : description du profil épidémiologique, clinique, thérapeutique et évolutif des intoxications aux organophosphorés

Méthodes : étude rétrospective concernant 62 dossiers de malades hospitalisés au service de pédiatrie 2 de l'hôpital d'enfants de Casablanca pour intoxications aux organophosphorés entre janvier 2000 et septembre 2006.

Résultats : les intoxications aux organophosphorés ont représenté 9,2\% de l'ensemble des intoxications avec une moyenne d'âge de 4,5 ans (extrêmes 2 mois-14 ans), sexe ratio : 1,03 .

L'intoxication a été accidentelle dans $81 \%$ des cas et volontaire dans $19 \%$ des cas. La voie de pénétration a été dominée par la voie digestive dans $91 \%$ des cas. $77,5 \%$ des cas ont consulté dans les 6 heures après l'ingestion du toxique avec un délai moyen de $3 \mathrm{~h} 35 \mathrm{~min}$ 
(extrêmes 20min-3 jours). La symptomatologie clinique a été dominée par des signes muscariniques avec hyper sialorrhée dans $14,5 \%$ des cas, un myosis dans $5 \%$ des cas, encombrement bronchique dans $5 \%$ des cas. Des signes nicotiniques : trémulations $5 \%$ des cas, convulsions 3\% des cas. 34\% des cas ont été asymptomatiques. Sur le plan thérapeutique $43,5 \%$ des malades ont bénéficié d'un lavage gastrique ; $19,35 \%$ ont reçu de l'atropine. L'évolution à court terme a été bonne dans la plupart des cas avec un délai d'hospitalisation qui varie de 24 à $48 \mathrm{~h}$. Un transfert en unités de soins intensifs a été fait dans 2 cas pour détresse neurologique et respiratoire. Tous les cas des intoxications volontaires ont été adressés à la consultation de pédopsychiatrie.

Conclusion : la prévention des intoxications aux organophosphorés paraît primordiale par le biais de l'information et de l'éducation de la population sur les risques des accidents domestiques chez l'enfant et sur les conditions de stockage des produits industriels à domicile ce qui permet de réduire la morbidité et la mortalité.

\section{Références :}

1. Sylla M. Intoxication aigue accidentelle chez l'enfant au service de pédiatrie de l'hôpital Gabriel Touré. Mali Médical. 2006 ; XXI(2).

2. Delvallée G. et coll. Étude hémodynamique des intoxications volontaires aiguës graves par insecticides organophosphorés (onze cas). Réanim. Soins Intensifs Méd. Urgence $1991 ; 7: 179-82$.

\section{Intoxications aux psychotropes chez l'enfant}

\section{A. ZAIDANI, A. FADIL, F. JENNANE, B. SLAOUI,} F. DEHBI

Service de pédiatrie 2, Hôpital d'enfants, CHU Ibn Rochd, Casablanca, Maroc

Introduction : les intoxications médicamenteuses constituent un motif fréquent de consultation aux urgences pédiatriques. Elles sont le plus souvent accidentelles mais parfois volontaires en particulier chez l'adolescent. L'évolution est souvent bénigne mais peut être parfois grave engageant le pronostic vital. L'intoxication aux psychotropes en constitue une part importante, en particulier celle aux anxiolytiques qui représente $25 \%$ des intoxications médicamenteuses (1).

Objectif : analyser le profil épidémiologique et clinique des intoxications aux psychotropes chez l'enfant.

Méthodes : étude rétrospective intéressant 159 dossiers de malades hospitalisés au service de Pédiatrie 2 de l'Hôpital d'enfants de Casablanca pour intoxication aigue aux psychotropes sur une période de 7 ans (Janvier 2000 à Septembre 2006).

Résultats : les intoxications aux psychotropes ont constitué $23.5 \%$ de l'ensemble des cas d'intoxications colligés au service, et $48.6 \%$ des intoxications médi- camenteuses. Elles sont dominées par les anxiolytiques mis en cause dans $69.8 \%$ des cas, suivis par les neuroleptiques rencontrés dans $13.2 \%$ des cas. Les intoxications aux barbituriques et aux antidépresseurs ont été notées dans $4.4 \%$ chacune, tandis que les antiépileptiques ont été incriminés dans $3.83 \%$ des cas. Les intoxications aux hypnotiques et aux morphiniques ont été observées respectivement dans $1.25 \%$ et $0.62 \%$ des cas, alors que $2.5 \%$ des patients ont présenté des intoxications mixtes. L'âge moyen de nos patients est de 5 ans [15 mois -15 ans].Le sex ratio est de 0.83 . L'intoxication est accidentelle dans $79 \%$, volontaire dans $17 \%$. Le délai moyen de consultation est de 5h18min [10 min - 48h]. Les signes cliniques sont dominés par les signes neurologiques observés dans $87.4 \%$ des cas, en particulier les troubles de conscience et de l'équilibre, suivis par les signes digestifs à type de douleurs abdominales et de vomissements dans 27.6 $\%$ des cas. Sur le plan thérapeutique, $46 \%$ des malades ont bénéficié d'un lavage gastrique, $9.4 \%$ des cas ont reçu un traitement symptomatique, tandis que $42.7 \%$ ont relevé de la simple surveillance. À noter que $8.1 \%$ des cas ont entrepris des mesures traditionnelles pour provoquer les vomissements : l'administration du lait dans $6.3 \%$ des cas, du Henné dans $1.25 \%$ des cas et du vinaigre dans $0.62 \%$ des cas. L'évolution a été favorable dans tous les cas, et la sortie des patients a eu lieu après éducation de la famille.

Conclusion : les intoxications aux psychotropes constitue une urgence médicale en pédiatrie pouvant mettre en jeu le pronostic vital, d'où l'intérêt d'une meilleure information du grand public et la prise en charge psychiatrique spécialisée dans les cas d'intoxications volontaires.

\section{Référence :}

1. Lavaud J. Intoxications aigues de l'enfant. EMC. $2002 ; 4: 125-\mathrm{A} 15$

\section{Intoxications aigues aux produits ména- gers chez l'enfant}

F. BELMOURIDA, S. SALIMI, F. JENNANE, B. SLAOUI, F. DAHBI

Service de pédiatrie 2 de l'hôpital d'enfants du CHU Ibn Rochd, Casablanca, Maroc

Objectif : description du profil épidémiologique, clinique, thérapeutique et évolutif des Intoxications aiguës infantiles aux produits ménagers.

Méthodes : étude rétrospective qui concernait 47 dossiers d'enfants admis pour intoxications aiguës aux produits ménagers au service de pédiatrie 2 de l'hôpital d'enfants du CHU Ibn Rochd de Casablanca durant la période du mois de janvier 2000 au mois de septembre 2006.

Résultats : ces intoxications représentent 5,9\% de l'ensemble des intoxications aiguës observées chez l'en- 
fant durant la période du mois de janvier 2000 au mois de septembre 2006

L'âge moyen : 3 ans et 8 mois (extrêmes : 2 mois-12 ans) ; le sexe ratio : 2,25 ; le produit ménager : Eau de javel $73 \%$; le délai moyen de prise en charge : $2 \mathrm{~h}$ 36min (extrêmes : $1 \mathrm{~h}-7 \mathrm{~h}$ ) ; les circonstances de survenue : accidentelles $96 \%$ avec un âge moyen de 1 an, volontaires $3,8 \%$ avec un âge moyen de 12 ans ; la symptomatologie clinique est dominée par les vomissements $19 \%$ et la toux $15 \%$; les examens complémentaires : la radiographie pulmonaire a été pratiquée dans $73 \%$ des cas et s'est révélée normale dans $10 \%$ des cas. La fibroscopie digestive a été faite dans $46 \%$ des cas et elle a mis en évidence une oesophagite avec gastrite modérée dans $5 \%$ des cas. Le traitement adopté a été la restriction alimentaire chez $15 \%$, la prescription d'antibiothérapie chez $15 \%$, de pansement gastrique chez $11 \%$. L'évolution à court terme a été marquée par la survie de tous les cas ; 7,6\% ont bénéficié d'une réanimation. L'évolution à long terme a été bonne dans tout les cas.

Conclusion : la prévention est primordiale par l'éducation et l'information des parents et de l'enfant, pour cela le personnel médical et paramédical, les éducateurs, les industriels, les organismes sociaux doivent diffuser par les moyens les plus divers et les plus simples l'information utile afin de diminuer ces intoxications

\section{Référence :}

1. Baad J. Conduite à tenir devant les intoxications les plus fréquentes. Urgences. EMC. 1991 ; 241-15A (60) Edition technique. Paris.

\section{Les intoxications aigues de l'enfant aux produits pétroliers et dérivés. A propos de 97 cas}

S. SALIMI, N. AMENZOUI, A. SAKHI, F. JANNANE, F. DEHBI

Service de Pédiatrie II, Hôpital d'enfants, CHU Ibn Rochd, Casablanca, maroc

Introduction : l'intoxication aigue de l'enfant est une cause fréquente d'hospitalisation des enfants, elle constitue un problème majeur de santé publique par la morbidité et la mortalité qui lui sont liées. L'intoxication aux produits pétroliers et leurs dérivés représente la seconde étiologie par ordre de fréquence soit $20,7 \%$ de toutes les intoxications aigues.

Objectifs : le but de notre travail est de préciser le profil épidémiologique, clinique et thérapeutique des intoxications aux produits pétroliers.

Matériels et méthodes : il s'agit d'une étude rétrospective de 97 observations colligées au service de Pédiatrie II de l'hôpital d'enfants de Casablanca, entre janvier 2000 et septembre 2006.

Résultats : l'intoxication aigue au produits pétroliers représente $12,3 \%$ de l'ensemble des intoxications aigues. L'âge moyen est de 2 ans et demi avec des extrêmes de 12 mois à 6 ans, sexe ratio : 0,5. Dans notre série, le diluant de peinture représente $63 \%$ des cas, l'essence $21 \%$, le butane $6 \%$ et le gasoil $2 \%$. Par ailleurs, dans 3 cas la nature du produit n'a pas été précisée. Le délai moyen de consultation est de 3 heures (20 min - 3 jours), l'intoxication est survenue accidentellement dans tous les cas. La symptomatologie clinique est dominée par des signes digestifs, de type vomissements, douleurs abdominales (50\%) et de signes neurologiques de l'obnubilation à la perte de connaissance (39\%), et des troubles respiratoires (toux, polypnée) $27 \%$ dont 2 cas de suffocation. La radio du thorax faite chez tous nos patients a relevé une pneumopathie d'inhalation dans $12 \%$ des cas. Le traitement est essentiellement symptomatique à base d'antibiothérapie (30 cas) et de pansements gastriques (17 cas). L'évolution était favorable dans $97 \%$ des cas. 5 patients ont été transférés en réanimation dans un tableau de détresse respiratoire et neurologique.

Conclusion : l'intoxication aux produits pétroliers peut être grave et engager le pronostic vital, d'où l'intérêt de la prévention qui doit être effectuée à grande échelle par la mise en place de cellules de réflexion regroupant des pédiatres, réanimateurs et médecins du centre antipoison et l'organisation de campagnes d'éducation des parents.

\section{Références :}

1. Baad J. Conduite à tenir devant les intoxications les plus fréquentes. Urgences. EMC 41-15A (60) Edition techniques. Paris 1991.

2. Huauth. J. Traitement des intoxications aiguës chez l'enfant. Ann. Nestlé 1990, 48 : 27-33.

3. Layaud J. et coll. Accidents et intoxications chez l'enfant. Pédiatrie-EMC 4127 A10 - 15A Edition technique. Paris 1982.

\section{Intoxication au métoclopramide}

\section{A. FADIL, A. ZAIDANI, F. JENNANE, B . SLAOUI, F. DEHBI}

Service de pédiatrie 2 ; Hôpital d'Enfants, Casablanca, Maroc

Introduction : l'intoxication au métoclopramide devient un motif de consultation de plus en plus fréquent en raison de sa large prescription dans les gastroentérites aigues infectieuses et dans le RGO. Elle se manifeste par des dystonies avec une dyskinésie faciale. Elle peut être la conséquence d'un surdosage ou d'intoxication accidentelle, mais aussi d'une susceptibilité particulière de l'organisme de l'enfant.

Objectif : évaluer le profil épidémiologique et clinique des intoxications au métoclopramide dans notre service.

Méthode : étude rétrospective concernant 35 dossiers médicaux des enfants hospitalisés dans notre service 
pour intoxication au métoclopramide durant la période de Janvier 2000 jusqu' au septembre 2006.

Résultat : l'intoxication au métoclopramide représente $5 \%$ de l'ensemble des intoxications de l'enfant, avec une moyenne d'age de sept ans et demi (extrême 3 mois - 13 ans), et un sexe ratio : 0,94. Dans 33 cas, les dyskinésies sont survenues suite à des doses thérapeutiques, et dans 3 cas, il s'agissait d'un surdosage ( 2 intoxications accidentelles et 1 volontaire).

L'installation des signes cliniques se fait en général dans les trois jours suivant l'ingestion du produit. Dans les cas de surdosage, le délai d'apparition des signes cliniques est immédiat survenant dans les cinq heures après l'ingestion ( 2 heures à 8 heures), et dans les cas de susceptibilité au métoclopramide, ce délai est variable de trois heures à trois jours. Les signes cliniques sont dominés par les dyskinésies faciales dans tous les cas, accompagnées de trismus et de torticolis dans huit cas, et une aphasie dans un seul cas. Sur le plan thérapeutique, 95,5\% des cas ont reçu des benzodiazépines (Valium $0,5 \mathrm{mg} / \mathrm{Kg}$ en intra rectal), et $8,5 \%$ ont reçu de l'Artane $(0,1 \mathrm{mg} / \mathrm{kg} / \mathrm{j})$. L'évolution était favorable dans tous les cas, avec un délai d'hospitalisation moyenne de 24 heures.

Conclusion : les dyskinésies neurologiques secondaires à la prise de métoclopramide, que ce soit à dose thérapeutique ou après un surdosage, sont en général de bon pronostic. Cependant, elles risquent d'entraver l'observance thérapeutique en cas d'indication justifiée, d'où l'intérêt de faire recours aux anti-émétiques à action périphérique dont les effets extra pyramidaux observés, sont extrêmement faibles.

\section{Référence :}

1. Lavaud J. Intoxications aigues de l'enfant. EMC.2002 ; 4 : 125-A15.

\section{Les intoxications aigues aux plantes aux urgences}

R. ANAFLOUS, A. SASSI, K. CHARAF, S. SOUROUR, AR. EL ADIB, H. NEJMI, S. YOUNOUS, MA. SAMKAOUI.

Service d'Anesthésie- Réanimation, CHU Mohammed VI, Marrakech, Maroc.

Des mots tels que "nature" ou "naturellement" ne sont pas forcément synonymes de sécurité. En effet, notre belle nature abrite toute une série de plantes toxiques qui peuvent être vénéneuses et hallucinogènes, capables de provoquer des symptômes graves même lorsqu'elles sont consommées en faible quantité, causant des perturbations des métabolismes des différents organes et pouvant provoquer la mort.

Selon les dires d'ethnopharmacologues et d'ethnobotanistes, les effets de substances naturelles hallucinogènes ont été utilisés partout dans le monde, comme médiateurs sacrés entre l'homme et l'au delà. Au $21^{\text {e }}$ siècle, des gourous électroniques transmettent des "recettes" via "Internet" à des non-initiés en quête de sensations fortes. Sans conscience tribale, ni crainte de poursuite légale, n'importe qui peut maintenant diffuser ses propres concoctions pour voyager vers un monde meilleur. Historiquement, les plantes toxiques ont également été largement utilisées à des fins criminelles.

Le Maroc possède une flore très variée et diversifiée (4200 espèce et sous-espèce). Il existe une vivacité des systèmes de soins traditionnels moins coûteux, plus prés de la population et mieux insérés dans le contexte social d'où la floraison de milliers de tradipraticiens qui utilisent les plantes comme principal moyen thérapeutique.

Les études médicales au Maroc ne comprennent pas l'étude poussée des plantes et de la toxicologie, ce qui fait qu'en plus des risques auxquels se soumettent les victimes, ce sont les retards de diagnostic qui posent des problèmes, simplement parce que le médecin consulté en urgence n'y pense pas ou n'a pas la connaissance nécessaire pour reconnaître la symptomatologie. Nous proposons à travers une revue des principales plantes responsables d'intoxications aigues au Maroc de dresser la symptomatologie clinique orientant vers le diagnostic et approcher les modalités de prise en charge aux urgences.

\section{Épidémiologie des intoxications aiguës aux urgences}

K. FATH, Y. HANAFI, J. ZIATI, A. ABBAD, AR. EL ADIB, H. NEJMI, S. YOUNOUS, M.A. SAMKAOUI

Service d'Anesthésie- Réanimation, CHU Mohammed VI, Marrakech, Maroc

Objectif : les intoxications aigues, causées par une exposition volontaire ou accidentelle à un ou plusieurs toxiques, constituent une part importante d'admissions aux urgences des sujets de moins de 30 ans. Le but de ce travail est d'analyser les caractéristiques épidémiologiques des intoxications aigues admises aux urgences.

Méthodes : étude rétrospective incluant tous les patients admis, en deux unités de soins intensifs des urgences de Marrakech, pour intoxication aigue à différents toxiques. Elle concerne 184 patients pour la

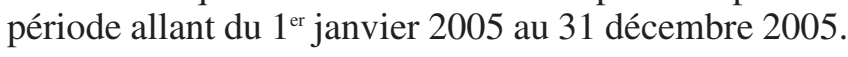

Les caractéristiques épidémiologiques ont été relevées à partir des dossiers médicaux des patients.

Résultats : les intoxications aigues représentent $20 \%$ des admissions. L'âge moyen de nos patients est de $27,5[ \pm 4,88]$ ans avec une nette prédominance féminine $(70 \%) .50 \%$ des patients sont âgés de 15 à 25 ans. La fréquence des intoxications volontaires est plus importante par rapport aux intoxications accidentelles $(82 \%)$. Le pic de survenue est la période estivale. 
L'intoxication médicamenteuse occupe la première place avec 55 cas $(29,8 \%)$, suivie par les intoxications aux organophosphorés avec 49 cas $(26,6 \%)$ puis l'intoxication au monoxyde de carbone (CO) avec 42 cas $(22,8 \%)$. Chez 24 patients (13\%), le toxique n'a pas pu être identifié. Les raisons de l'intoxication volontaire sont à la fois complexes et multiples. Le facteur le plus souvent rapporté par le malade ou son entourage est le conflit familial, problèmes sentimentaux, échec scolaire. Le délai de prise en charge est de $12 \pm 6$ heures. Le transport a été non médicalisé dans tous les cas. La ventilation assistée a été instaurée chez 10 patients $(5,4 \%)$ avec une durée moyenne de 10 jours. Le taux de mortalité a été de $6,5 \%$.

Commentaires : les intoxications aigues constituent toujours une préoccupation d'actualité pour les équipes médicales des services d'accueil des urgences, c'est une situation pathologique fréquente. Elles intéressent un ensemble de patients dont les caractéristiques épidémiologiques sont relativement stables depuis plusieurs années (population jeune, majoritairement de sexe féminin). La réussite du traitement des intoxications aigues passe par un enchaînement bien conduit des réflexes de maintien des fonctions vitales, d'une analyse des signes cliniques et paracliniques et de l'indication d'un traitement spécifique et épurateur en appoint au traitement symptomatique. Dans notre étude, le bilan toxicologique n'a jamais pu être réalisé et aucun patient n'a reçu de traitement antidotique spécifique. L'amélioration de la prise en charge de nos patients doit passer obligatoirement par le développement des recherches toxicologiques dans nos établissements ainsi que l'utilisation d'antidotes dans l'arsenal thérapeutique, mais surtout par l'acheminement rapide des patients, les campagnes d'information, l'application de protocoles adaptés et le suivi psychiatrique des patients.

\section{Prévalence des effets indésirables médi- camenteux à l'hôpital d'enfants : étude transversale}

M. AGOUZAL ${ }^{(2)}$, R. BENKIRANE(1), S. ACHOUR ${ }^{(1)}$, R. BENJELLOUN ${ }^{(1)}$, A. TEBAA ${ }^{(1)}$, N. SMIRES ${ }^{(1)}$, S. SKALLI ${ }^{(1)}$, G. BENABDALLAH ${ }^{(1)}$, L. ALJ ${ }^{(1)}$, N. MESBAHI ${ }^{(1)}$, A. SOULAYMANI ${ }^{(2)}$, R. SOULAYMANI ${ }^{(1,3)}$

(1) Centre Antipoison et de Pharmacovigilance, Rabat ;

(2) Laboratoire de pharmacologie et toxicologie, Kenitra ;

(3) Faculté de médecine et de pharmacie, Rabat, Maroc.

Introduction : la culture de notification des EIM (effets indésirables médicamenteux) doit être implantée dans notre milieu médical. La stratégie du Centre Antipoison et de Pharmacovigilance du Maroc (CAPM) est articulée sur la collaboration du CAPM avec les services hospitaliers dans le cadre de la sécurité du patient. Ainsi, une étude a été effectuée à l'hôpital d'enfants de Rabat dont l'objectif était d'identifier la prévalence, la nature et la gravité des Effets Indésirables des produits de santé dans le milieu hospitalier.

Méthodes : une étude prospective de 5 jours du 20/06/2005 au 25/06/2005 a été menée sur les enfants hospitalisés pour moins ou plus de 24 heures. Cette étude a pu être réalisée grâce à la collaboration active des médecins et du personnel infirmier de l'HER. Elle s'est déroulée dans les services de médecine et de réanimation, le bloc médical et le bloc porte ainsi qu'au niveau du laboratoire d'hématologie.

Résultats : parmi les 380 patients hospitalisés durant la période, 22 ont présenté au moins un effet indésirable médicamenteux, soit une prévalence de $5.8 \%$ (IC 95\% : $3.45 ; 8.15$ ). Pour 10 patients $(45.4 \%)$ les EIM ont provoqué ou prolongé l'hospitalisation. Dans 59\% des cas, il s'agissait d'un EIM grave. La comparaison du groupe qui a développé un EIM avec la population totale hospitalisée durant la période d'études a montré que les garçons étaient concernés les plus. La prévalence due à un EI ou prolongation de l'hospitalisation est de 2.6\%. Le taux des EI graves était de 2,9\%.

Conclusion : cette étude a permis de sensibiliser les professionnels de santé et a démontré que la pathologie inhérente à l'utilisation des médicaments n'était pas négligeable à l'hôpital. D'où la nécessité de mettre en place une politique de gestion de ce risque à l'échelle de chaque établissement hospitalier.

\section{Évaluation de la méthode buprénorphi- ne CEDIA. Problèmes liés à l'interféren- ce des opiacés}

H. KHEROUF, F. PARANT, E. SEIDEL, S. CAVORET, M. MOULSMA

Laboratoire de Pharmacologie-Toxicologie-Éléments Traces (UF 21.303), Fédération de Biochimie., Hôpital Édouard Herriot, Lyon, France.

Introduction : autorisée en France depuis 1996, prescrite à 85000 patients dépendants à l'héroïne, le succès de la buprénorphine haut dosage (Subutex®) a un revers : son détournement avec un important trafic (20\% à $25 \%$ du Subutex ${ }^{\circledR}$ est sniffé ou injecté par les usagers et $4 \%$ à $5 \%$ trafiqué). Quelques cas de décès imputables à l'association Subutex ${ }^{\circledR}$ - benzodiazépines sont reportés. Dans notre laboratoire, le dosage de la buprénorphine est réalisé par GC-MS ou LC-MS. Nous avons évalué une technique immunoenzymatique CEDIA de dosage de la buprénorphine urinaire totale avec pour double objectif de pouvoir répondre aux situations d'urgence et de dégager du temps technique.

Matériel et méthode : la méthode CEDIA est réalisée sur un analyseur Cobas Mira en mode semi-quantitatif 
(cinq calibrateurs de 0 à $75 \mu \mathrm{g} / \mathrm{L}$ ) selon les recommandations du fabriquant (lecture photométrique à 550 $\mathrm{nm})$. Seule une auto-dilution au tiers par réduction de prise d'essai est ajoutée. Les résultats sont comparés à la GC-MS. Les urines testées sont celles du personnel du laboratoire (urines témoins) $(n=44)$ et celles de patients majoritairement suivis par un Centre de Soin Spécialisé aux Toxicomanes (CSST) $(\mathrm{n}=140)$.

Résultats : validation analytique : la méthode CEDIA est à la fois juste sur des charges préparées à partir de solutions Promochem ${ }^{\circledR}$ et précise (Répétabilité $<6 \%$ pour l'ensemble des points de gamme; $\mathrm{n}=20$ Reproductibilité à $14,2 \%$ et $7,7 \%$ pour des contrôles ciblés à $7 \mu / \mathrm{L}$ et $19 \mu / \mathrm{L} ; n=20)$. La linéarité de la méthode de 0 à $75 \mu \mathrm{g} / \mathrm{L}$ a été vérifiée selon la procédure idoine du logiciel MultiQC®. La limite de détection analytique $((1 \mu \mathrm{g} / \mathrm{L})$ est largement inférieure au seuil de positivité de $5 \mu \mathrm{g} / \mathrm{L}$ proposé par Böttcher (1). Enfin, il apparaît nécessaire d'étalonner à chaque série. Interférence avec les opiacés : Conformément aux observations de Pavlic (2), la présence d'opiacés dans les urines en concentrations élevées « positive » la méthode buprénorphine CEDIA. Un seuil de positivité de $20 \mu \mathrm{g} / \mathrm{L}$ (courbes ROC) peut être proposé en présence d'opiacés, $($ sensibilité $=78 \%$; spécificité $=$ $100 \%$ ). En l'absence d'opiacés, un seuil de $5 \mu \mathrm{g} / \mathrm{L}$ est raisonnable (sensibilité $=94 \%$; spécificité $=100 \%)$. Quantification sur des urines de patients : la représentation de Bland-Altman et la regression de PassingBablok montrent une bonne concordance des résultats entre la méthode CEDIA et la GC-MS. Cas clinique : nous rapportons le cas d'une intoxication accidentelle par Subutex ${ }^{\circledR}$ chez une enfant de 15 mois pour laquelle les dosages de buprénorphine urinaire ont été de $200 \mu \mathrm{g} / \mathrm{L}$ par CEDIA et $207 \mu \mathrm{g} / \mathrm{L}$ par LC-MS (norbuprénorphine : $226 \mu \mathrm{g} / \mathrm{L}$ ).

Conclusion : en pratique quotidienne, l'interférence liée aux opiacés est fréquemment rencontrée sur des urines de patients d'un CSST et impose d'utiliser un seuil de positivité supérieur à $5 \mu \mathrm{g} / \mathrm{L}$. La méthode CEDIA apparait plus particulièrement adaptée aux situations d'urgence qu'aux suivis de compliance.

\section{Références :}

1. Böttcher M. Evaluation of buprenorphine CEDIA assay versus GC-MS and ELISA using urine samples from patients in substitution treatment. J. Anal. Toxicol. $2005 ; 29$ : 769-76.

2. Pavlic M. et coll. Cross-reactivity of the CEDIA buprenorphine assay with opiates: an austrian phenomenon? Int. J. Legal Med. 2005 ; 119 : 378-81.

\section{Analyse du phostoxin. Un cas d'intoxica- tion mortelle}

M.A. BELLIMAM, A. STAMBOULI, M. BENAMMI, A. EL BOURI, T. BOUAYOUN.

Laboratoire de Recherches et d'Analyses Techniques et Scientifiques - Gendarmerie Royale Rabat, Maroc

Objectifs : le phostotoxin (AlP) est disponible sans restriction au Maroc. Il est utilisé comme taupicide et aussi comme agent fumigeant à l'égard des insectes ravageurs des céréales entreposés. En cas d'ingestion orale, le phosphure réagit avec l'eau et l'acidité stomacale pour produire de l'hydrogène phosphoré $\left(\mathrm{PH}_{3}\right)$, gaz hautement toxique dégageant une odeur alliacée, qui n'est perceptible dans l'air qu'à partir de concentrations de 0,002 pour mille (1).

La présente étude vise la mise au point d'une méthode analytique pour la caractérisation du phostoxin à l'état brut et sa détection dans les liquides biologiques.

Méthodes : la caractérisation du produit commercial est réalisée par spectrophotométrie Infra rouge et fluorescence $\mathrm{X}$, alors que la détection de l'hydrogène phosphoré résultant de l'hydrolyse de AlP est conduite en chromatographie gazeuse couplée à la spectrométrie de masse en mode espace de tête. L'intoxication au phostoxin est confirmée par analyse en spectrophotométrie IR du contenu gastrique après filtration, mais aussi par détermination par spectrophotométrie d'absorption atomique des teneurs en aluminium et phosphore après minéralisation. A cet effet, 1,5 $\mathrm{g}$ du contenu gastrique est minéralisé dans un four micro-ondes à une température de $200{ }^{\circ} \mathrm{C}$ pendant 30 minutes en présence d'un mélange de $7 \mathrm{~mL}$ d'acide nitrique concentré et de $1 \mathrm{~mL}$ de peroxyde d'hydrogène $\left(\mathrm{H}_{2} \mathrm{O}_{2}\right)$. Le minéralisat est repris par $50 \mathrm{~mL}$ d'eau distillée puis analysé en spectrophotométrie d'absorption atomique (mode four électrothermique pour le dosage du phosphore et mode flamme protoxyde d'azote-acétylène pour le dosage d'aluminium).

Résultats : cette méthode a été appliquée au cas d'une intoxication mortelle d'un homme de 27 ans, cultivateur dont le corps a été retrouvé dans un champ de petits pois. A proximité du cadavre, a été découvert un flacon renfermant une poudre grisâtre. L'analyse par spectrophotométrie d'absorption atomique du minéralisat du contenu gastrique de la victime a conduit à la détermination de fortes teneurs d'aluminium $(17000 \mu \mathrm{g} / \mathrm{g})$ et de phosphore $(3240 \mu \mathrm{g} / \mathrm{g})$ comparés aux valeurs usuelles ( $\mathrm{Al}: 10 \mu \mathrm{g} / \mathrm{g} ; \mathrm{P}: 430 \mu \mathrm{g} / \mathrm{g}$ ), ce qui est synonyme d'une intoxication au phostoxin.

Conclusion : compte tenu de la forte volatilité de l'hydrogène phosphoré, produit d'hydrolyse du phostoxin, le présent travail propose une méthode alternative faisant concourir d'une part, la spectrophotométrie IR pour la caractérisation du produit brut et d'autre part, la spectrophotométrie d'absorption atomique pour l'évaluation des teneurs en aluminium et phosphore dans le contenu gastrique.

\section{Référence :}

1. Anger F. et coll. Intoxication fatale par ingestion de phosphure d'aluminium. Toxicorama. $1999 ; 11$ : 245-9. 


\section{Analyse de la paraphénylènediamine (PPD). Application aux cas d'intoxica- tions}

\author{
M.A. BELLIMAM, A. STAMBOULI, N. EL KARNI,
} T. BOUAYOUN, A. EL BOURI

Laboratoire de Recherches et d'Analyses Techniques et Scientifiques de la Gendarmerie Royale, Rabat, Maroc

Objectif : la paraphénylènediamine (PPD) est un colorant utilisé dans l'industrie (teinturerie, cosmétique, développement photographique, fabrication des pneus). Au Maroc, elle trouve son application dans la coloration des cheveux chez les femmes et la teinture de fourrures animales. Les données statistiques du centre anti-poison du Maroc rapportent que la PPD est responsable de $25 \%$ des intoxications attribuées aux produits de pharmacopée traditionnelle. La femme jeune est la plus touchée $(75,4 \%)$ surtout lors de tentatives d'avortement ou d'autolyse. Le décès survient dans plus de $30 \%$ des cas. Les rares méthodes analytiques décrites dans la littérature, consacrées à la caractérisation de la PPD dans les fluides biologiques, sont peu sensibles et exigent d'importantes prises d'essai (1) souvent indisponibles. Le présent travail propose une méthode optimisée de déproténeïsation (2), d'extraction et d'analyse GC/MS de la PPD à partir de quantités d'urines et de contenus gastriques ne dépassant guère $2 \mathrm{~mL}$.

Méthodes : cette méthode optimise un protocole de déproténeïsation, d'extraction et de dérivatisation par l'anhydride trifluoroacétique (TFA) de la PPD. $1 \mu \mathrm{L}$ de l'extrait dérivatisé est analysé en GC/MS sur une colonne capillaire HP $5(25 \mathrm{~m}$ x $0.2 \mathrm{~mm} \times 0.11 \mu \mathrm{m})$ avec un programme de température $\left(50^{\circ} \mathrm{C}, 2 \mathrm{~min}\right.$; $\left.15^{\circ} \mathrm{C} / \mathrm{min} ; 280^{\circ} \mathrm{C}, 10 \mathrm{~min}\right)$. La benzidine est employée comme étalon interne pour le dosage du produit.

Résultats : après caractérisation de la PPD par les techniques spectroscopiques IR, RMN ${ }^{1} \mathrm{H},{ }^{13} \mathrm{C}$ et $\mathrm{GC} / \mathrm{MS}$ (3), une optimisation de la détection du produit a été conduite en GC/MS après dérivatisation au TFA et a permis d'atteindre une limite de quantification équivalente à $1 \mathrm{ng} / \mathrm{L}$. Appliquée à un cas d'intoxication mortelle d'une jeune femme de 21 ans morte par insuffisance respiratoire, cette méthode a permis la détermination d'une teneur de $0,25 \mathrm{mg} / \mathrm{L}$ dans les urines. De même, qu'elle a contribué à élucider une intoxication aiguë d'une jeune fille de 14 ans, qui a été sauvée après intubation et traitement. Dans ce cas, la quantité de la PPD présente dans les urines a été évaluée à $0.26 \mu \mathrm{g} / \mathrm{L}$.

Conclusion : alors que les techniques colorimétriques usuellement employées nécessitent des prises d'essai de $150 \mathrm{~mL}$ d'urines ou d'autres analytiques utilisent des quantités de $126 \mathrm{~g}$ de contenu gastrique (1), cette nouvelle méthode permet la détection de traces de la PPD et réduit considérablement les prélèvements des fluides biologiques $(2 \mathrm{~mL})$.

\section{Références :}

1. Pufal E. et coll. Determination of postmortem blood and gastric contents. Heppenheim, Verlag Dr Dieter Helm. 2001 ; S262-7.

2. Stambouli H. et coll. Optimization of an analytical method for detecting paraphenylenediamine (PPD) by GC-MS-ion trap in biological liquids. Forensic Sci. Int. 2004 ; 146S : 87-92.

3. Bellimam My A. et coll. Analysis of paraphenylenediamine. Acta. Clin. Belgica. 2006 ; 61 : S41-7.

\section{Intoxication à la fluméquine (Apurone ${ }^{\circledR)}$ et interférence spectrale avec les dépis- tages urinaires des stupéfiants par tech- nique immunoenzymatique EMIT II Plus. À propos d'un cas}

\section{F. PARANT, M. MOULSMA}

Laboratoire de Pharmacologie-Toxicologie-Éléments Trace (UF 21.303), Fédération de Biochimie, Hôpital Édouard Herriot, Lyon, France

Observation : une femme de 21 ans ingère dans un moment de dépression consécutive à une conjugopathie environ 15 comprimés d'Apurone ${ }^{\circledR}$ (soit $6 \mathrm{~g}$ de fluméquine, 5 fois la dose thérapeutique). À son arrivée aux urgences environ 4 heures après la prise, un état confusionnel et une agitation (Glasgow 13) sont observés. L'examen clinique retrouve également une tachycardie à 155 bpm et une tachypnée à 30/35 cycles / min. Sur le plan biologique, seuls sont à noter des bicarbonates à $12 \mathrm{mmol} / \mathrm{L}(\mathrm{N}=24-26 \mathrm{mmol} / \mathrm{L})$ et une $\mathrm{PCO} 2$ à $2.4 \mathrm{kPa}$ $(\mathrm{N}=4.9-5.7 \mathrm{kPa})$. L'évolution clinique a été rapidement favorable et sans séquelle. La patiente étant connue pour une consommation de substances illicites, un dépistage urinaire de stupéfiants est demandé.

Interférences : ce dépistage comprenant la recherche des opiacés, cocaïne, cannabis et amphétaminiques n'a pas été possible en raison d'une interférence avec la technique immunoenzymatique EMIT II Plus (automatisée sur analyseur Dimension) produisant les messages d'erreurs analytiques suivants : NEG err'A' haute (pour OPI et COC) et $>$ dom.calcul (pour AMPH et THC). L'étude des données brutes (DATA) objective une interférence spectrale en raison d'une forte absorbance en début de réaction à $340 \mathrm{~nm}$ (mesure de la réduction du co-facteur NADP en NADPH $\mathrm{H}^{+}$). L'analyse des urines par chromatographie liquide couplée à une barrette de diode (LC-DAD) retrouve la présence de fluméquine et de ses métabolites caractérisés par un coefficient d'extinction molaire élevé à $340 \mathrm{~nm}$ $\left(\varepsilon=6039 \mathrm{~L} \cdot \mathrm{mol}^{-1} . \mathrm{cm}^{-1}\right.$ à $\left.\mathrm{pH} 5,5\right)$. Une dilution des urines au demi a permis de corriger l'interférence (recherches négatives) au prix d'un doublement des seuils de positivité. Les investigations concernant les stupéfiants n'ont pas été poursuivies. Enfin, l'interférence spectrale a été reproduite sur des urines de 
témoins chargées en fluméquine (poudre Sigma) lorsque la concentration en cette molécule atteint $2,5 \mathrm{~g} / \mathrm{L}$.

Discussion : la fluméquine (Apurone ${ }^{\circledR}$ ) est une quinolone fluorée de $1^{\text {ère }}$ génération habituellement bien tolérée. Le tableau clinique d'une intoxication sévère est caractérisé par des troubles neurologiques et métaboliques (acido-cétose et hyperglycémie) (1). Environ $63 \%$ de la dose ingérée est éliminée dans les urines en 24h sous forme de fluméquine, de 7-hydrofluméquine et de dérivés glucuroconjugués (2). Cette interférence est intéressante à double titre : (a) les interférences spectrales liées à la présence de médicaments sont finalement assez rares car nécessitant la conjonction d'un coefficient d'extinction molaire élevé à la longueur d'onde de mesure et de fortes concentrations (3) ; (b) les interférences liées aux quinolones décrites jusqu'à lors sont de type « réaction croisée ». Ainsi, un traitement par ofloxacine positive une recherche d'opiacés en EMIT (4).

\section{Références :}

1. Cochard G. et coll. À propos d'un cas d'intoxication à la fluméquine. Conv. Méd. 1984 ; 5 : 439-40.

2. Schuppan D. et coll. Plasma and urine levels of flumequine and 7-hydroxyflumequine following single and multiple oral dosing. J. Antimicrob. Chemother. $1985 ; 15: 337-43$.

3. Joseph R. et coll. Interference by nonsteroidal antiinflammatory drugs in EMIT and TDx assays for drugs of abuse. J. Anal. Toxicol. 1995 ; 19 : 13-7.

4. Baden L. R. et coll. Quinolones and false-positive urine screening for opiates by immunoassay technology. JAMA 2006 ; $286: 3115-9$.

\section{Takaout, quelle teneur en paraphénylène diamine ?}

Y. BOUSLIMAN ${ }^{(1)}$, T. BASSET ${ }^{(1)}$, J.P. GAYMONTCHAMP(1) $^{(1)}$ A.A. ZEGGWAGH ${ }^{(2)}$, Y. CHERRAH $^{(3)}$, M. OLLAGNIER ${ }^{(1)}$

(1) Laboratoire de Pharmaco-Toxicologie, Hôpital Bellevue, CHU Saint Etienne, France ;

(2) Réanimation médicale et toxicologie clinique, Hôpital Avicenne, CHU Rabat Salé, Maroc ;

(3) Laboratoire de Pharmaco-Toxicologie, Faculté de Médecine et de Pharmacie, Rabat, Maroc.

Objectif : la paraphénylène diamine (PPD), amine aromatique, est un produit minéral hautement toxique utilisé comme teinture noire pour les cheveux et comme adjuvant au henné (1). Au Maroc, elle est disponible sous forme de roche en vente libre chez les herboristes connue sous le nom de takaout roumia (2). L'objectif de ce travail a consisté à analyser la teneur en paraphénylène diamine de cette roche.

Méthode : le dosage de la paraphénylène diamine aux seins de trois échantillons différents de takaout roumia a été basé sur la technique de la chromatographie en phase gazeuse couplée à la spectrométrie de masse.

Résultats : l'analyse de la teneur en PPD des échantillons a révélé un taux de $90.9 \pm 0.5 \%$ pour l'échantillon A, de $84.65 \pm 4.65 \%$ pour l'échantillon B et de $9.69 \pm 1.54 \%$ pour l'échantillon C.

Conclusion : l'analyse de différents échantillons de takaout roumia nous a montré que la teneur en PPD de cette roche est différente d'un échantillon à l'autre selon sa source d'approvisionnement (3).

\section{Références :}

1. Arditti J. et coll. Takaout, teinture capillaire naturelle ? Bull. Méd. Lég. Toxicol. 1980 ; 23.

2. Elomari M. et coll. Relation entre la dose et l'atteinte myocardique induite par la paraphénylène diamine chez le rat. J. Prat. $2004 ; 14: 117$.

3. Munday R. et coll. Muscle necrosis by N-Methyled p-phenylen diamines in rats : structure-activity relationships and correlated with free-radical production in vitro. Toxicology 1989 ; 57(3) : 303-14.

\section{L'influence de la pollution nitrique sur la santé de la population de la zone M'NASRA (Région du Gharb Chrarda Beni Hssen)}

\section{IDRISSI ALAMI $^{(2)}$, M.ADDOU $^{(1)}$, A.SOULAYMANI $^{(2)}$, M. ZERAOULI ${ }^{(3)}$}

(1) Laboratoire d'Optoélectronique et de Physico Chimie des Matériaux ;

(2) Laboratoire de Génétique et Biométrie, Faculté des Sciences, Kenitra ;

(3) Laboratoire des analyses d'eau et sol de l'Office régional de la mise en valeur agricole, kenitra, Maroc

Objectif : l'objectif de notre étude est d'évaluer la qualité des eaux souterraines de la zone M'NASRA, par l'analyse de trois paramètres : nitrates, salinité et $\mathrm{pH}$ au niveau de 161 puits.

Résultats : les résultats du dosage des nitrates effectué sur les eaux des puits de cette région, montrent que les moyennes générales de la concentration des nitrates en 1993 et 2003 sont respectivement de $106.4 \mathrm{mg} / \mathrm{L}$ et de $119.6 \mathrm{mg} / \mathrm{L}$; soit une augmentation de $12.4 \%$. En $1993,27.3 \%$ des puits répondaient aux normes de potabilité et contenaient moins de $50 \mathrm{mg} / \mathrm{L}$ de nitrates. Dix années plus tard (en 2003) il n'y a plus que $17.4 \%$ des puits qui répondaient aux normes de potabilité. Concernant le potentiel d'hydrogène des eaux souterraines, les mesures montrent que les moyennes générales du pH en 1993 et 2003 sont respectivement de 7.49 et de 7.55 ; il ressort de ces résultats que le $\mathrm{pH}$ des eaux des puits de la région étudiée M'NASRA est relativement stable, et légèrement basique. Quant à la conductivité électrique moyenne des eaux des puits mesurée à $20^{\circ} \mathrm{C}$ pour les eaux des 161 puits, elle est respectivement de $0.85 \mathrm{mS} / \mathrm{cm}$ et de $1.09 \mathrm{mS} / \mathrm{cm}$ pour 
les années 1993 et 2003.

Conclusion : nos résultats montrent que les eaux souterraines de la zone M'NASRA se sont salinisées et présentent un taux de nitrates de plus en plus élevé entre 1993 et 2003 et constituent de ce fait une nuisance pour l'irrigation et pour le consommateur.

\section{Fiche de données de sécurité (FDS)}

\section{B. LAARAJ, M. BOUDOUANE, S. CHARIOUI,} A. EL KHOLTI

Service de santé au travail, CHU Ibn Rochd, Casablanca, Maroc

Objectif : sensibiliser les professionnels de santé au travail sur l'intérêt de la fiche de données de sécurité dans la prévention des risques chimiques en milieu du travail.

Méthodes : il existe environ 16 millions de substances chimiques dans le monde, selon le Chemical Abstracts Service (CAS). Plus de 100000 substances chimiques sont enregistrées et commercialisées en Europe, dont $10 \%$ vendues en quantités supérieures à 10 tonnes. Plus de $20 \%$ des substances chimiques produites en grandes quantités au sein de l'UE sont dépourvues de données toxicologiques. Ce n'est donc pas parce qu'un produit est commercialisé qu'il est sans danger. Ainsi la prévention des risques chimiques s'articule, pour l'essentiel, autour d'un système basé sur l'information des utilisateurs des produits chimiques dangereux. Certes, cette information ne suffit pas à elle seule à mettre sous contrôle les risques chimiques vu la diversité avec laquelle ils peuvent s'exprimer, mais elle constitue un maillon essentiel. De tous les outils qui permettent aux utilisateurs de ces produits de préserver leur santé et leur sécurité. Cette information passe de manière incontournable par la fiche de données de sécurité qui permet d'établir des fiches ou notices aux postes du travail.

Conclusion : dans ce travail nous rappelons les principaux éléments constituant une fiche de données de sécurité et sa place dans des risques chimiques en milieu du travail.

\section{Maîtrise de la qualité à propos de deux cas de soumission chimique}

\section{PERRIN}

Institut de Recherche Criminelle de la Gendarmerie Nationale, Rosny-sous-Bois, France

Introduction : la maîtrise de la qualité des résultats analytiques rendus est la préoccupation majeure de tout laboratoire. L'assurance qualité est un élément primordial pour donner confiance dans les résultats transmis aux requérants. Cependant, le processus qualité mis en place au laboratoire en vue de notre prochaine accréditation, est tributaire de paramètres extérieurs non mầtrisés, que l'on peut rassembler sous la notion de "critères pré-analytiques". En effet le site de prélèvement, la quantité prélevée, la température de conservation des échantillons avant l'analyse peuvent influencer les résultats. Cette problématique de prélèvements non conformes sera abordée à partir de deux dossiers traités récemment au département toxicologie de l'IRCGN. L'IRCGN appartient au réseau européen de sciences criminelles (ENFSI). Il s'implique depuis de nombreuses années dans la démarche assurance qualité, ce qui devrait aboutir à terme à l'accréditation d'une partie des activités. Si l'activité au laboratoire est sous contrôle, il existe des paramètres que le laboratoire ne maîtrise pas. Il s'agit de la qualité des échantillons transmis au laboratoire. En effet le site de prélèvement sanguin, les matrices prélevées, la quantité d'échantillon transmise au laboratoire, le conditionnement, la température de conservation des échantillons avant l'analyse peuvent influencer les résultats.

Cas cliniques : Cas n $^{\circ} 1$ "des substances volatilisées": Melle A, lors de ses vacances passées dans le sud-ouest de la France, disparaît le 25 août vers minuit. Elle est retrouvée inanimée puis transportée au CHU. Seule une alcoolémie (par méthode enzymatique) est réalisée sur le prélèvement pratiqué vers $5 \mathrm{~h}$ du matin $\left(2,72 \mathrm{~g}\right.$. $\left.\mathrm{L}^{-1}\right)$. Elle est autorisée à quitter l'hôpital en fin de matinée, mais elle ne porte plainte que le même jour vers $14 \mathrm{~h}$ à la brigade de gendarmerie. Lors de son arrivée aux urgences médico-judiciaires seul un prélèvement sanguin est réalisé vers $15 \mathrm{~h}$, mais sur un tube non conforme (tube avec gel séparateur de cellules, absence de conservateur et d'inhibiteurs de la glycolyse, $3 \mathrm{ml}$ de sang dans un tube de $10 \mathrm{ml}$ ). Le médecin décide de ne pas prélever d'urines. Les scellés sont transmis par voie postale à température ambiante. Cas $\mathrm{n}^{\circ} 2$ "la preuve par l'oeuf" : M. D, administre à son épouse et à son insu, des comprimés de Noctamide® (lormétazépam) dissous dans une boisson, puis l'agresse sexuellement. Le lendemain, pris de remords, il se présente spontanément à la brigade de gendarmerie afin que l'on localise son épouse (en déplacement professionnel hors de la ville) pour qu'elle se dirige vers l'hôpital le plus proche afin qu'elle fasse retirer le corps étranger introduit la veille par M. D. Une procédure est ouverte pour viol, mais seuls des prélèvements de sang sont pratiqués sur madame D. Aucun prélèvement d'urine n'est demandé par le médecin. L'ensemble des scellés est transmis par voie postale (à température ambiante).

Méthode : ces deux dossiers sont soumis aux analyses prévues par la stratégie analytique du département.

Résultats : $\underline{\operatorname{Cas} n^{\circ} 1}$ la recherche des substances volatiles a montré des traces d'éthanol (valeur inférieure à la limite de quantification). Il a par ailleurs été mis en évidence de l'acide 11 nor delta 9 tetrahydrocannabinolique et du 11 hydroxy tétrahydrocannabinol. Cas $\mathrm{n}^{\circ} 2$ : des traces de lormétazépam, ont été mises en évidence dans le sang de Mme D.

Discussion : ${\operatorname{Cas~}{ }^{\circ} 1}^{1}$ : la disparition en 12 heures de la totalité de l'éthanol peut être expliquée par la conjonc- 
tion de trois paramètres : métabolisation, volatilisation d'une partie de l'éthanol dans le volume mort au dessus du liquide au cours des opérations de décongélation, adsorption de l'éthanol sur le gel séparateur. Confrontée aux résultats, Melle A. indique qu'elle a consommé du cannabis la veille des faits vers $17 \mathrm{~h}$. Le GHB a été recherché mais (ainsi que la pharmacocinétique le laissait prévoir) une concentration physiologique à $0,5 \mathrm{mg}$ par $\mathrm{mL}$ de sang a été mise en évidence. Cas $\mathrm{n}^{\circ} 2$ : le lormétazépam a été mis en évidence malgré un délai très long entre la prise de xenobiotiques et le prélèvement, et malgré une température de conservation inadaptée. Remarque : dans les deux cas, le Parquet n'a pas souhaité faire prélever des cheveux, matrice alternative qui aurait permis, le cas échéant de mettre en évidence une exposition isolée à un xenobiotique.

Conclusion : quels que soient les efforts produits dans la chaîne de l'assurance qualité, le laboratoire reste tributaire de paramètres pré-analytiques qu'il ne peut pas maîtriser Deux cas cliniques reçus récemment au département toxicologie viennent démontrer l'importance de la qualité du prélèvement.

\section{Usage de cannabis et autres drogues licites et illicites chez les adolescents de Rabat en scolarisation}

$\underline{\text { S. JAYCHE }}{ }^{(1)}$, S. SKALLI ${ }^{(2)}$, R. SOULAYMANI BENCHEIKH $^{(2,3)}$, A. MOKHTARI ${ }^{(1)}$, A. SOULAYMANI ${ }^{(1)}$

(1) Laboratoire de génétique et biométrie, Faculté des Sciences de Kénitra ;

(2) Centre Anti-poison et de Pharmacovigilance du Maroc ;

(3) Faculté de Médecine et de Pharmacie, Rabat, Maroc.

Introduction : le cannabis indica ou chanvre indien est la drogue illicite la plus répandue dans le monde. L'évaluation de l'usage du cannabis chez les jeunes, a une grande importance dans l'élaboration des stratégies de lutte et de prévention contre la consommation de cette drogue.

Objectif : l'objectif de notre étude est de s'informer sur les fréquences et les modes d'usage de cannabis chez les adolescents: déterminer la fréquence de l'usage du cannabis et maâjoun; établir une relation entre l'usage du cannabis et maâjoun et les conditions socioéconomiques du consommateur.

Méthode : au cours de l'année 2006 on a réalisé une enquête par questionnaire anonyme de 43 questions concernant les données épidémiologiques, socio-économiques et l'usage de drogues, auprès de 248 élèves de troisième année secondaire de 8 lycées différents.

Résultats : on a évalué la fréquence des usagers et les non usagers de drogues. Quatre-vingt huit pour cent (88\%) des élèves sont des non usagers, et douze pour cent $(12 \%)$ des usagers. Au sein des $12 \%$ des consom- mateurs de drogues (licites et illicites), la majorité (90\%) est de sexe masculin. L'analyse de variance a montré qu'il n'y a aucune influence de niveau de vie sur la consommation ou sur la nature de drogue prise $(\mathrm{p}=0.6)$.

Conclusions : dans notre étude préliminaire, l'abus de cannabis et/ou maâjoun, touche principalement le sexe masculin. En revanche le niveau de vie des élèves n'influence pas la consommation.

\section{Références :}

1. Wone. I. et coll. Prévalence de l'usage du cannabis en milieu étudiant à Dakar. Santé 2004 ; 14(1) : 49-53.

\section{L'envenimation scorpionique. A propos de 85 cas}

N. AMENZOUI, H. SAMLAK, S. SALIMA, F. JENNANE F. DEHBI

Service de Pédiatrie II, Hôpital d'enfant, CHU Ibn Rochd, Casablanca, Maroc

Introduction : l'envenimation scorpionique est un accident fréquent au Maroc, elle représente un véritable problème de santé publique par la morbidité et la mortalité qui lui sont liées. Son incidence est estimée à 40.000 cas par an.

Objectif : le but de notre travail est de préciser les aspects cliniques, thérapeutiques, évolutifs et préventifs de cette pathologie.

Méthode : il s'agit d'une étude rétrospective de 85 observations colligées au service de Pédiatrie II de l'Hôpital d'enfant de Casablanca sur une période de 7 ans, de janvier 2000 à septembre 2006.

Résultats : l'envenimation scorpionique représente $10,87 \%$ de l'ensemble des intoxications aigues de l'enfant. L'âge moyen est de 7 ans avec des extrêmes de 9 mois à 14 ans et un sexe ratio de 0,5 . Dans notre série $30 \%$ des patients sont originaires de la région de Khouribga, 20\% de Béni Mellal, 15\% de Casablanca et régions, $5 \%$ d'El Kalaa et 30\% représenté par le reste des régions du sud du Maroc .le délais moyen de consultation est de 10 heures. L'espèce noire de scorpion est responsable de $40 \%$ des piqûres, l'espèce jaune de $11 \%$ des piqûres et dans $55 \%$ des cas l'espèce n'a pas été précisée. Sur le plan clinique $47 \%$ des patients ont présenté seulement des signes locaux à l'endroit de la piqûre (œdème, rougeur et douleur), $53 \%$ des patients ont présenté des manifestations systémiques de type hypersudation (36\%), vomissement (29\%), troubles de conscience (16\%), priapisme (13\%), frisson $(10 \%)$ et état de choc (5\%). Le traitement est essentiellement symptomatique à base d'antalgiques dans tous les cas, d'antiémétiques dans 24 cas, d'hydratation dans 45 cas, d'antihypertenseurs dans 10 cas, et dans 13 cas on a eu recours aux dopaminergiques. L'évolution était favorable dans $85 \%$ des cas, par ailleurs $15 \%$ des patients ont été transférés en réanima- 
tion dans un tableau de détresse respiratoire et hémodynamique.

Conclusion : l'envenimation scorpionique peut être grave et engager le pronostic vital à brève échéance par ses complications cardio-vasculaires, respiratoires et neurologiques. Le pronostic dépend de la précocité de la prise en charge, de l'âge des patients et du type de scorpion. La prévention reste le seul moyen efficace pour réduire l'incidence des envenimations scorpioniques et ceci par l'éducation des parents et des enfants, la formation du personnel médical et paramédical à ce type d'envenimation en collaboration avec le centre antipoison et de pharmacovigilance.

\section{Références :}

1. Abouyala.B. Envenimation scorpionique à la province de Tiznit. Thèse Médecine, Casablanca 1998 N4 4 .

2. Abroug F. et coll. Envenimation scorpionique : Aspects cliniques, physiopathologiques et thérapeutiques. Réanimation Urgences. 2000 ; 9 : 45-50.

3. Soulaymani R. et coll. Piqures de scorpions : état actuel des connaissances. Espérances Médicales. 1999 ; 5(51) : 291-6.

\section{Intoxication au benzène : à propos d'un cas}

B. LAARAJ, S. CHARIOUI, M. BOUDOUANE, A. EL KHOLTI

Service de santé au travail, CHU Ibn Rochd, Casablanca, Maroc

Objectif : sensibiliser les professionnels de santé au travail sur les risques liés au benzène en milieu du travail.

Méthodes : le benzène largement utilisé dans l'industrie comme intermédiaire de synthèse. Il est absorbé par toutes les voies d'exposition et rapidement distribué, préférentiellement dans les tissus riches en lipides, le cerveau, le sang, les reins et le foie. Sa métabolisation a lieu principalement dans le foie ainsi que dans la moelle osseuse. Le Centre international de recherche sur le cancer a conclu qu'il y a suffisamment de signes de cancérogénicité du benzène chez l'humain.

Observation : Mr B.A., âgé de 52 ans salarié dans une multinationale de fabrication de pneumatique depuis une vingtaine d'année, sans antécédents pathologiques particuliers a présenté après 15 ans d'activité une hypoplasie médullaire confirmée par un examen anatomopathologique. L'interrogatoire professionnel retrouve la notion de manipulation du benzène sans aucun moyen de protection individuelle ni collective. Après la confirmation du diagnostic un reclassement professionnel a été décidé à un poste de gardien en plein air. L'examen clinique retrouve un patient en assez mauvais état général, avec des lésions desquamatives au niveau palmo-plantaire et une thrombopénie.

Conclusion : la toxicité du benzène n'est plus à démontrer, ainsi des efforts de sensibilisation des salariés, des employeurs et des professionnels de santé au travail nécessite de mettre en place des mesures de prévention adaptées pour maîtriser le risque chimique en milieu du travail.

\section{Étude des piqûres et des envenimations scorpioniques au niveau de Khouribga (2001 à 2005)}

O.K. TAMIM ${ }^{(1)}$, R. SOULAYMANI-BENCHEIKH ${ }^{(2,3)}$

A. SOULAYMANI (1), M. TAYEBI ${ }^{(1)}$, I. SEMLALI ${ }^{(2)}$, G. EL OUFIR ${ }^{(2)}$, A. MOKHTARI ${ }^{(1)}$

(1) Faculté des Sciences, Université Ibn Tofail, Kénitra ;

(2) Centre Anti-Poison et de Pharmacovigilance du Maroc ;

(3) Faculté de Médecine et de Pharmacie de Rabat.

Objectif : connu pour sa biodiversité scorpionique, le Maroc, principalement son centre sud, est le lieu de piqûres fréquentes et d'envenimations sévères. La présente étude vise est la mise à jour de la situation épidémiologique des piqûres et des envenimations scorpioniques au niveau de la délégation de Khouribga afin d'évaluer les risques d'intoxications scorpioniques, d'améliorer la prise en charge et de diminuer le taux de morbidité et de mortalité à partir d'un système d'information.

Méthode : une étude, effectuée à partir des registres de la délégation médicale de Khouribga, a permis de suivre les indicateurs de morbidité et de mortalité durant cinq années. Elle compte 6011 cas de piqûres et d'envenimations scorpioniques.

Résultats : la fréquence des piqûres et des envenimations scorpioniques est importante durant la période estivale avec un pic au mois de juillet, principalement entre 18 et 24 heures. La moyenne d'âge des piqués est de 26,80 \pm 0,27 ans, dont le tiers sont des enfants d'âge 15 ans. Le sex-ratio (M/F) est de 0.95 . Le pourcentage de piqués arrivant avant 1 heure à la structure sanitaire est de $55 \%$ et $13 \%$ des piqués n'ont pas été traités. Le taux d'envenimation est de $8.1 \%$ et le taux de létalité de $0.6 \%$. L'analyse de la variance montre que l'âge, la classe d'admission et le temps post-piqûre sont déterminants pour l'évolution des patients piqués. Le nombre de cas envenimés a augmenté de 2001 à 2003, puis il a légèrement diminué de 2003 à 2005 parallèlement à l'incidence. Le pourcentage de patients traités a diminué par contre, ceux référés et hospitalisés ont augmenté avec quelques légères fluctuations. Le taux de létalité générale et le taux de létalité par envenimation ont diminué d'année en année avec cependant une importante augmentation en 2005.

Conclusion : cette étude a donc révélé une importante sensibilisation de la population et une nette amélioration de la conduite à tenir. Cependant, la morbidité et la létalité des piqués sont encore loin d'être négligeables. 


\section{Les facteurs de gravité des intoxications oxycarbonées au Maroc (1994-2004)}

S. SELLAMI ${ }^{(1)}$, A. SOULAYMANI ${ }^{(1)}$, M. IDRISSI ${ }^{(2)}$, A. MOKHTARI ${ }^{(1)}$, R. SOULAYMANI-BENCHEIKH ${ }^{(2,3)}$

(1) Laboratoire de génétique et biométrie - Faculté des Sciences de Kénitra ;

(2) Centre Anti-Poison et de Pharmacovigilance du Maroc (CAPM) ;

(3) Faculté de Médecine et de Pharmacie de Rabat.

Objectifs : le monoxyde de carbone (CO) est la première cause de mortalité dans le monde, ce tueur silencieux est responsable de plusieurs décès par an dans notre pays, ainsi que de plusieurs milliers d'intoxications entraînant hospitalisations et dans certains cas, des séquelles irréversibles.

Notre travail s'inscrit dans le cadre d'une approche épidémiologique ayant pour objectif d'évaluer les caractéristiques épidémiologiques et cliniques de l'intoxication oxycarbonée au Maroc, de déterminer les facteurs de gravité pouvant influencer le pronostic vital de l'intoxiqué et de se positionner par rapport aux données internationales ; ce qui servirait de base pour permettre la mise en place des moyens de prévention adaptés.

Méthode : notre étude rétrospective porte sur 5072 cas d'intoxications oxycarbonées déclarés au CAPM, depuis janvier 1994 jusqu'à décembre 2004. L'analyse statistique a consisté en un test $\chi^{2}$, avec analyse de variance à un facteur et le calcul du risque relatif (RR).

Résultats : l'âge moyen des intoxiqués est de 25.67 et le sexe ratio est de 0.42 . Il s'agit d'intoxication presque toujours accidentelle revêtant un caractère collectif dans $46 \%$ des cas, elle survient surtout à domicile (87\%). Une nette recrudescence est relevée pendant la période hivernale ( $73 \%$ des déclarations). La population urbaine est plus concernée avec un taux de $82 \%$. La région du centre est la plus touchée (20\%), suivie de la région $\mathrm{du}$ nord ouest (13.65\%). Le délai de consultation montre une corrélation significative avec l'évolution de l'état du patient. L'apparition de signes neurovégétatifs $(\mathrm{p}<0.001)$, cutanés $(\mathrm{p}<0.05)$ et/ou cardiovas-culaires $(\mathrm{p}<0.05)$, influence considérablement l'évolution de l'intoxication, avec des RR respectifs de 9.02, 3.65, et de 2.58. Le taux de létalité générale est de $0.53 \%$ avec un age moyen de décès de $29.83 \pm 3.39$ ans.

Conclusion : compte tenu de l'importance des intoxications oxycarbonées qui surviennent chaque année au Maroc, des mesures de leur prévention doivent être entreprises et leur prise en charge restent encore à améliorer. 